ESAIM: M2AN 48 (2014) 1473-1494

DOI: $10.1051 / \mathrm{m} 2 \mathrm{an} / 2014006$
ESAIM: Mathematical Modelling and Numerical Analysis

www.esaim-m2an.org

\title{
ROBUST OPERATOR ESTIMATES AND THE APPLICATION TO SUBSTRUCTURING METHODS FOR FIRST-ORDER SYSTEMS
}

\author{
Christian Wieners ${ }^{1}$ And Barbara Wohlmuth ${ }^{2}$
}

\begin{abstract}
We discuss a family of discontinuous Petrov-Galerkin (DPG) schemes for quite general partial differential operators. The starting point of our analysis is the DPG method introduced by [Demkowicz et al., SIAM J. Numer. Anal. 49 (2011) 1788-1809; Zitelli et al., J. Comput. Phys. 230 (2011) 2406-2432]. This discretization results in a sparse positive definite linear algebraic system which can be obtained from a saddle point problem by an element-wise Schur complement reduction applied to the test space. Here, we show that the abstract framework of saddle point problems and domain decomposition techniques provide stability and a priori estimates. To obtain efficient numerical algorithms, we use a second Schur complement reduction applied to the trial space. This restricts the degrees of freedom to the skeleton. We construct a preconditioner for the skeleton problem, and the efficiency of the discretization and the solution method is demonstrated by numerical examples.
\end{abstract}

Mathematics Subject Classification. 65N30.

Received April 25, 2013. Revised January 12, 2014.

Published online August 13, 2014.

\section{INTRODUCTION}

Petrov-Galerkin schemes for the numerical solution of partial differential equations are among the most flexible and powerful discretization techniques. In particular, these methods allow for different test and ansatz spaces in the variational problem formulation. This additional flexibility possibly increases the robustness and stability in case of parameter dependent systems. Uniform stability can be granted for a large class of partial differential equations including, e.g., the nearly incompressible linear elasticity case. However, the choice of suitable finite dimensional test and trial spaces is far from trivial in the conforming setting since uniform infsup conditions have to be satisfied. Recently introduced discontinuous Petrov-Galerkin schemes $[15,16,18,34]$ are of special interest and provide attractive alternatives to standard conforming schemes, see, also e.g., $[9,13,17]$.

Here we focus on this newly introduced abstract family of discretizations and provide robust a priori estimates and define iterative solvers. More precisely, we show that a broad class of linear partial differential equations can be efficiently approximated by methods which allow for a local reduction to positive definite Schur complement problems. Schur complement reductions are extensively investigated for finite element methods of least-squares type $[4,5]$ and, recently, for least-square problems on element level resulting in discontinuous Petrov-Galerkin

\footnotetext{
Keywords and phrases. First-order systems, Petrov-Galerkin methods, saddle point problems.

1 Institut für Angewandte und Numerische Mathematik, KIT, Karlsruhe, Germany. christian.wieners@kit.edu

2 Fakultät Mathematik M2, Technische Universität München, Garching, Germany. wohlmuth@ma.tum.de
} 
methods $[15,16]$. In combination with the reduction to the interface $[10,25,27]$ efficient iterative solvers can be designed. Note that there is also a close link to the FOSLS scheme [1,2,11,22], to the Trefftz method (see [21] for an overview), and to a least-squares approach in negative norms for first-order systems [7].

The starting point is a linear first-order partial differential system. This is obtained either directly from balance equations and constitutive laws, e.g., in the case of Darcy's law, or alternatively we rewrite a second order equation as first order system by introducing auxiliary unknowns. To discretize this system, we use element-wise domain decomposition techniques involving traces, fluxes and suitable lifting operators. Once the traces on the element boundaries are known, the interior values can be reconstructed approximately by solving local low dimensional problems.

Before we consider the abstract setting, we sketch the ideas for a simple model problem in Section 2. The abstract setting for the DPG method fits perfectly well into the standard saddle point framework. In order to obtain optimal a priori bounds and to explain the concept of Petrov-Galerkin discretizations with optimal test spaces and the connection to Schur complements, we briefly review saddle point problems in Section 3. Then in Section 4, we verify the stability for several examples including elasticity, the Stokes system, the Helmholtz problem, and Maxwell's equation. In Section 5, it is shown that the stability of the skeleton reduction only depends on the stability constant of the first-order system. The discretization of the skeleton reduction is analyzed in Section 6 within the abstract framework. Finally in Section 7, we conclude with some numerical examples demonstrating the robustness of the method.

\section{A SCALAR MODEL PROBLEM}

We consider the second order elliptic partial differential equation

$$
-\Delta p+b \cdot \nabla p+a p=f \quad \text { in } \Omega, \quad p=0 \quad \text { on } \partial \Omega
$$

with $f \in \mathrm{L}_{2}(\Omega)$. The domain $\Omega \subset \mathbb{R}^{d}, d=2,3$, is assumed to be bounded and Lipschitz. Here $b \in \mathrm{H}^{1}\left(\Omega, \mathbb{R}^{d}\right)$ is the solution of an incompressible flow problem with $\nabla \cdot b=0$, and $a \in \mathrm{L}_{2}(\Omega)$ is a non-negative function. The regularity assumptions on $a$ and $b$ will guarantee that our weak formulation is well-defined. To define a first-order system, we introduce a flux variable $\sigma=b p-\nabla p$ and rewrite $(2.1)$ as $a p+\nabla \cdot \sigma=f$. Then the model equation reads as: find $\left(\sigma^{\mathrm{sol}}, p^{\mathrm{sol}}\right) \in U=\mathrm{H}(\operatorname{div}, \Omega) \times \mathrm{H}_{0}^{1}(\Omega)$ such $L\left(\sigma^{\mathrm{sol}}, p^{\mathrm{sol}}\right)=(0, f)$, with

$$
L(\sigma, p)=(\sigma-b p+\nabla p, a p+\nabla \cdot \sigma) .
$$

\subsection{Local substructuring}

Following the ideas of non-overlapping domain decomposition techniques, the discretization scheme is based on a disjoint partitioning $\bar{\Omega}=\bigcup_{\tau \in \mathcal{T}} \bar{\tau}$ into open subdomains $\tau \subset \Omega$. Let $\Gamma_{\mathcal{T}}=\bigcup_{\tau \in \mathcal{T}} \partial \tau$ be the skeleton of this decomposition. Then Schur complement techniques allow to eliminate the inner subdomain degrees of freedom and to reduce the system to trace values at the skeleton.

On each subdomain $\tau$, we define the local spaces $V_{\tau}=\mathrm{H}(\operatorname{div}, \tau) \times \mathrm{H}^{1}(\tau)$, on the boundaries $\partial \tau$ the local trace spaces $\hat{V}_{\tau}=\mathrm{H}^{-1 / 2}(\partial \tau) \times \mathrm{H}^{1 / 2}(\partial \tau)$, and the local trace operators $\gamma_{\tau}: V_{\tau} \longrightarrow \hat{V}_{\tau}$ with $\gamma_{\tau}(\sigma, p)=\left(\left.\sigma\right|_{\partial \tau} \cdot n_{\tau},\left.p\right|_{\partial \tau}\right)$, where $n_{\tau}$ is the outer normal vector on $\partial \tau$. We define the adjoint trace operator $\gamma_{\tau}^{\text {ad }}(\eta, q)=\left(\left.q\right|_{\partial \tau},\left.\eta\right|_{\partial \tau} \cdot n_{\tau}\right)$ and the adjoint differential operator

$$
L^{\mathrm{ad}}(\eta, q)=(\eta-\nabla q, a q-b \cdot \eta-\nabla \cdot \eta) .
$$

Then integration by parts yields for all test functions $(\eta, q) \in V_{\tau}$

$$
\begin{aligned}
(L(\sigma, p),(\eta, q))_{\tau} & =(\sigma-b p+\nabla p, \eta)_{\tau}+(a p+\nabla \cdot \sigma, q)_{\tau} \\
& =(\sigma, \eta-\nabla q)_{\tau}+(p, a q-b \cdot \eta-\nabla \cdot \eta)_{\tau}+\left\langle\left.\sigma\right|_{\partial \tau} \cdot n_{\tau},\left.q\right|_{\partial \tau}\right\rangle+\left\langle\left.\eta\right|_{\partial \tau} \cdot n_{\tau},\left.p\right|_{\partial \tau}\right\rangle \\
& =\left((\sigma, p), L^{\mathrm{ad}}(\eta, q)\right)_{\tau}+\left\langle\gamma_{\tau}(\sigma, p), \gamma_{\tau}^{\mathrm{ad}}(\eta, q)\right\rangle,
\end{aligned}
$$


where $\langle\cdot, \cdot\rangle$ denotes a vectorial duality pairing of $H^{-1 / 2}(\partial \tau)$ and $H^{1 / 2}(\partial \tau)$. Combining the local traces defines for $(\sigma, p) \in \mathrm{H}(\operatorname{div}, \Omega) \times \mathrm{H}^{1}(\Omega)$ the skeleton values

$$
\gamma_{\Gamma}(\sigma, p)=\left(\gamma_{\tau}(\sigma, p)\right)_{\tau \in \mathcal{T}} \in \hat{V}=\prod_{\tau \in \mathcal{T}} \hat{V}_{\tau}
$$

This gives for the solution $\left(\sigma^{\mathrm{sol}}, p^{\mathrm{sol}}\right) \in U$ of the system $L\left(\sigma^{\mathrm{sol}}, p^{\mathrm{sol}}\right)=(0, f)$ and its trace $\gamma_{\Gamma}\left(\sigma^{\mathrm{sol}}, p^{\mathrm{sol}}\right) \in \hat{V}$ the variational equality

$$
\left(\left(\sigma^{\mathrm{sol}}, p^{\mathrm{sol}}\right), L^{\mathrm{ad}}(\eta, q)\right)_{\tau}+\left\langle\gamma_{\tau}\left(\sigma^{\mathrm{sol}}, p^{\mathrm{sol}}\right), \gamma_{\tau}^{\mathrm{ad}}(\eta, q)\right\rangle=(f, q)_{\tau}, \quad(\eta, q) \in V_{\tau} .
$$

Restricting the test space $V=\prod_{\tau \in \mathcal{T}} V_{\tau}$ to the kernel of the adjoint operator

$$
\mathcal{N}\left(L^{\mathrm{ad}}\right)=\left\{(\eta, q) \in V: L^{\mathrm{ad}}(\eta, q)=0\right\},
$$

we observe that the skeleton values $\gamma_{\Gamma}\left(\sigma^{\text {sol }}, p^{\text {sol }}\right)$ satisfy

$$
\sum_{\tau}\left\langle\gamma_{\tau}\left(\sigma^{\mathrm{sol}}, p^{\mathrm{sol}}\right), \gamma_{\tau}^{\mathrm{ad}}(\eta, q)\right\rangle=(f, q)_{\Omega}, \quad(\eta, q) \in \mathcal{N}\left(L^{\mathrm{ad}}\right) .
$$

Knowing $\gamma_{\Gamma}\left(\sigma^{\text {sol }}, p^{\text {sol }}\right)$, we can recover $\left(\sigma^{\text {sol }}, p^{\text {sol }}\right)$ by solving independently for each $\tau \in \mathcal{T}$ a local problem. Now the main idea is to define suitable discrete approximation spaces for $\gamma_{\Gamma}(U)$ and $\mathcal{N}\left(L^{\text {ad }}\right)$ such that the discrete version of (2.5) is well-defined. We point out that the spaces $\gamma_{\Gamma}(U)$ and $\mathcal{N}\left(L^{\text {ad }}\right)$ have a completely different structure. The kernel $\mathcal{N}\left(L^{\text {ad }}\right)$ is purely subdomain-wise defined. Thus $\mathcal{N}\left(L^{\text {ad }}\right)$ can be written as a product space on the subdomains, whereas each $(\sigma, p) \in U$ with a non-trivial trace $\gamma_{\Gamma}(\sigma, p)$ has at least two subdomains as support. Thus a discretization of $\gamma_{\Gamma}(U)$ and $\mathcal{N}\left(L^{\text {ad }}\right)$ will naturally lead to a Petrov-Galerkin scheme.

\subsection{A Petrov-Galerkin discretization}

We approximate the trace of the solution $\hat{u}^{\mathrm{sol}}=\gamma_{\Gamma}\left(\sigma^{\mathrm{sol}}, p^{\mathrm{sol}}\right)$ of $(2.5)$ in a given discrete space $\hat{U}_{h} \subset \gamma_{\Gamma}(U)$. A natural choice of $\hat{U}_{h}$ is to take the trace of the fluxes of mixed finite elements, such as, e.g., Raviart-Thomas or BDM elements, see, e.g., [8], for the first component, and for the second component the traces of standard conforming finite elements. The Petrov-Galerkin solution $\hat{u}_{h}^{\text {sol }}=\left(\hat{u}_{\tau, h}^{\text {sol }}\right) \in \hat{U}_{h}$ of $(2.5)$ is defined by

$$
\sum_{\tau}\left\langle\hat{u}_{\tau, h}^{\mathrm{sol}}, \gamma_{\tau}^{\mathrm{ad}} v_{h}\right\rangle=\left((0, f), v_{h}\right)_{\Omega}, \quad v_{h} \in \mathcal{N}_{h} .
$$

To obtain a well-defined system, the choice of $\mathcal{N}_{h}$ is crucial. Is the dimension of $\mathcal{N}_{h}$ too small compared to the dimension of $\hat{U}_{h},(2.6)$ has no unique solution. Is the dimension too large, then there is possibly no solution. To guarantee a unique solution of (2.6), the construction of $\mathcal{N}_{h}$ is done in two steps. Firstly, we choose finite element spaces $H_{\tau, h} \subset \mathrm{L}_{2}\left(\tau, \mathbb{R}^{d} \times \mathbb{R}\right)$ and $V_{\tau, h} \subset V_{\tau}$ such that the dimension of the discrete kernel $\mathcal{N}\left(L_{h}^{\text {ad }}\right)=\prod_{\tau} \mathcal{N}_{\tau}\left(L_{h}^{\text {ad }}\right)$ with

$$
\mathcal{N}_{\tau}\left(L_{h}^{\mathrm{ad}}\right)=\left\{v_{\tau, h} \in V_{\tau, h}:\left(L^{\mathrm{ad}} v_{h, \tau}, u_{h, \tau}\right)_{\tau}=0 \text { for } u_{h, \tau} \in H_{\tau, h}\right\}
$$

is larger or equal than the dimension of $\hat{U}_{h}$. Secondly, we select a positive definite, continuous and symmetric bilinear form $a_{\tau}(\cdot, \cdot)$ on $V_{\tau, h} \times V_{\tau, h}$ and define for $\hat{u}_{h} \in \hat{U}_{h}$ the solution $N_{\tau}\left(\hat{u}_{h}\right) \in \mathcal{N}_{\tau}\left(L_{h}^{\text {ad }}\right)$ by

$$
a_{\tau}\left(N_{\tau}\left(\hat{u}_{h}\right), v\right)=\left\langle\hat{u}_{\tau, h}, \gamma_{\tau}^{\text {ad }} v\right\rangle, \quad v \in \mathcal{N}_{\tau}\left(L_{h}^{\text {ad }}\right) .
$$

We point out that $N_{\tau}\left(\hat{u}_{h}\right)$ is, by construction, well-defined. It can also be obtained by the solution of the following local saddle point problem: find $\left(N_{\tau}\left(\hat{u}_{h}\right), M_{\tau}\left(\hat{u}_{h}\right)\right) \in V_{\tau, h} \times H_{\tau, h}$ such that

$$
\begin{aligned}
a_{\tau}\left(N_{\tau}\left(\hat{u}_{h}\right), v\right)+\left(M_{\tau}\left(\hat{u}_{h}\right), L^{\mathrm{ad}} v\right)_{\tau} & =\left\langle\hat{u}_{\tau, h}, \gamma_{\tau}^{\mathrm{ad}} v\right\rangle, & & v \in V_{\tau, h}, \\
\left(L^{\mathrm{ad}} N_{\tau}\left(\hat{u}_{h}\right), w\right)_{\tau} & =0, & & w \in H_{\tau, h} .
\end{aligned}
$$


If the pairing $\left(V_{\tau, h}, H_{\tau, h}\right)$ satisfy a suitable inf-sup condition, a unique solution exists of (2.8). Otherwise the first solution component is unique but not the second one. Associated with $N_{\tau}$ is the global operator $N_{h}$ which maps $\hat{U}_{h}$ into $\mathcal{N}\left(L_{h}^{\text {ad }}\right)$ by $\left.N_{h}\left(\hat{u}_{h}\right)\right|_{\tau}=N_{\tau}\left(\hat{u}_{h}\right)$. Now we set $\mathcal{N}_{h}=N_{h}\left(U_{h}\right)$. Obviously, this choice guarantees the existence of a solution of (2.6). Uniqueness is obtained if $\operatorname{dim} \mathcal{N}_{h}=\operatorname{dim} U_{h}$ or equivalently if the operator $N_{h}$ is injective. By construction, we find $\operatorname{dim} \mathcal{N}_{h} \leq \operatorname{dim} U_{h}$ and if $\operatorname{dim} \mathcal{N}_{h}=\operatorname{dim} U_{h}$, then (2.6) is equivalent to the symmetric positive definite variational problem: find $\hat{u}_{h}^{\text {sol }} \in \hat{U}_{h}$ such that

$$
\sum_{\tau} a_{\tau}\left(N_{h}\left(\hat{u}_{h}^{\mathrm{sol}}\right), N_{h}\left(\hat{u}_{h}\right)\right)=\left((0, f), N_{h}\left(\hat{u}_{h}\right)\right)_{\Omega}, \quad \hat{u}_{h} \in \hat{U}_{h} .
$$

The variational problem (2.9) is not very well suited for a direct numerical realization since it requires the knowledge of the action of the operator $N_{h}$ on each element of $\hat{U}_{h}$. Alternatively, combining (2.7) and (2.8), we can obtain the solution of $(2.5)$ by the solving the saddle point formulation: find $\left(v_{h}^{\text {sol }}, u_{h}^{\text {sol }}, \hat{u}_{h}^{\text {sol }}\right) \in \prod_{\tau} V_{\tau, h} \times$ $\prod_{\tau} H_{\tau, h} \times \hat{U}_{h}$ such that

$$
\begin{aligned}
a\left(v_{h}^{\mathrm{sol}}, v\right)+\left(u_{h}^{\mathrm{sol}}, L^{\mathrm{ad}} v\right)_{\Omega}-\left\langle\hat{u}_{h}^{\mathrm{sol}}, \gamma_{\Gamma}^{\mathrm{ad}} v\right\rangle & =0, & & v \in \prod_{\tau} V_{\tau, h}, \\
\left(L^{\mathrm{ad}} v_{h}^{\mathrm{sol}}, w\right)_{\Omega} & =0, & & w \in \prod_{\tau} H_{\tau, h}, \\
\left\langle\hat{u}, \gamma_{\Gamma}^{\mathrm{ad}} v_{h}^{\mathrm{sol}}\right\rangle & & =\left((0, f), N_{h}(\hat{u})\right)_{\Omega}, & \hat{u} \in \hat{U}_{h},
\end{aligned}
$$

where $a(v, w)=\sum_{\tau} a_{\tau}\left(\left.v\right|_{\tau},\left.w\right|_{\tau}\right), v, w \in \prod_{\tau} V_{\tau, h}$, and $\left\langle\cdot, \gamma_{\Gamma}^{\text {ad }} \cdot\right\rangle$ and $\left(L^{\text {ad }}, \cdot\right)_{\Omega}$ have to be understood in the broken subdomain-wise sense. By construction, (2.10) has a solution for all possible choices of $V_{\tau, h}, H_{\tau, h}$ and $\hat{U}_{h}$. However uniqueness is not granted. Under the assumption $\operatorname{dim} \mathcal{N}_{h}=\operatorname{dim} U_{h}$, uniqueness of $\hat{u}_{h}$ is given.

\section{SADDLE POINT ESTIMATES IN OPERATOR DEPENDENT NORMS}

It is shown in ([30], Thm. 3) for the Stokes problem and in [9] for Friedrichs' systems that an a priori estimate for the variational problem (2.4) can be derived from a single stability constant, and in ([20], Thm. 2.1) it is proved that the upper bound for the error of the discrete DPG method relies only on a local inf-sup constant. Optimal estimates for Petrov-Galerkin discretizations using Kato's identity on idempotent operators are derived in [33], and optimal estimates for saddle point problems are recently presented in [26]. These estimates depend on the considered norms, and thus smaller constants can be obtained by selecting proper norms. It is obvious that in the well-posed elliptic case with a symmetric bilinear form, the ellipticity and continuity constants are one if the norm is defined as energy norm. Here, we show that the concept of energy norms can be extended to the saddle point setting.

Let $X$ and $Y$ be Hilbert spaces, and let $A \in \mathcal{L}\left(X, X^{\prime}\right)$ be a positive and self-adjoint operator, so that $\|x\|_{A}=\sqrt{\langle A x, x\rangle}$ is a norm in $X$. Moreover, let $B \in \mathcal{L}\left(Y, X^{\prime}\right)$ be an injective operator and assume that the Schur complement $S=B^{\prime} A^{-1} B$ defines a norm in $Y$ by

$$
\|y\|_{S}=\sqrt{\langle S y, y\rangle}=\sup _{x \in X} \frac{|\langle B y, x\rangle|}{\|x\|_{A}}, \quad y \in Y .
$$

It is easy to see that if $B$ is continuous and the system is inf-sup stable, then the Schur complement norm is equivalent to the Hilbert space norm in $Y$. We now study in $W=X \times Y$ the saddle point operator

$$
K:=\left(\begin{array}{cc}
A & B \\
B^{\prime} & 0
\end{array}\right)=\left(\begin{array}{cc}
\operatorname{id}_{X} & 0 \\
B^{\prime} A^{-1} & \operatorname{id}_{Y}
\end{array}\right)\left(\begin{array}{cc}
A & 0 \\
0 & -S
\end{array}\right)\left(\begin{array}{cc}
\operatorname{id}_{X} & A^{-1} B \\
0 & \operatorname{id}_{Y}
\end{array}\right) .
$$

This block decomposition motivates us to define the self-adjoint and positive definite operator

$$
\widetilde{K}:=\left(\begin{array}{cc}
\operatorname{id}_{X} & 0 \\
B^{\prime} A^{-1} & \operatorname{id}_{Y}
\end{array}\right)\left(\begin{array}{cc}
A & 0 \\
0 & S
\end{array}\right)\left(\begin{array}{cc}
\operatorname{id}_{X} & A^{-1} B \\
0 & \operatorname{id}_{Y}
\end{array}\right)=\left(\begin{array}{cc}
A & B \\
B^{\prime} & 2 S
\end{array}\right)
$$


and the norm $\|(x, y)\|_{\widetilde{K}}^{2}=\langle\widetilde{K}(x, y),(x, y)\rangle$ in $W$. Observing the identity $K^{\prime} \widetilde{K}^{-1} K=\widetilde{K}$, we obtain

$$
\|K(x, y)\|_{\widetilde{K}^{-1}}^{2}=\left\langle K(x, y), \widetilde{K}^{-1} K(x, y)\right\rangle=\langle\widetilde{K}(x, y),(x, y)\rangle=\|(x, y)\|_{\widetilde{K}}^{2}
$$

for all $(x, y) \in W$, i.e., $\|K\|_{\mathcal{L}\left(W, W^{\prime}\right)}=\left\|K^{-1}\right\|_{\mathcal{L}\left(W^{\prime}, W\right)}=1$ with respect to the operator dependent norm in $W$.

Let $X_{h} \subset X$ and $Y_{h} \subset Y$ be a family of finite dimensional subspaces, and let $A_{h} \in \mathcal{L}\left(X_{h}, X_{h}^{\prime}\right)$ and $B_{h} \in \mathcal{L}\left(Y_{h}, X_{h}^{\prime}\right)$ be the Galerkin approximations of $A$ and $B$, respectively. The corresponding discrete Schur complement is given by $S_{h}=B_{h}^{\prime} A_{h}^{-1} B_{h}$. We assume uniform inf-sup stability of the discrete saddle point problem, i.e., there exists a $1 \geq \beta_{0}>0$ independent of $h$ such that

$$
\sup _{x_{h} \in X_{h}} \frac{\left|\left\langle B_{h} y_{h}, x_{h}\right\rangle\right|}{\left\|x_{h}\right\|_{A}} \geq \beta_{0} \sup _{x \in X} \frac{\left|\left\langle B y_{h}, x\right\rangle\right|}{\|x\|_{A}}, \quad y_{h} \in Y_{h} .
$$

This implies that the discrete operator $K_{h}\left(x_{h}, y_{h}\right)=\left(A_{h} x_{h}+B_{h} y_{h}, B_{h}^{\prime} x_{h}\right)$ is invertible, and we have the spectral bounds

$$
\beta_{0}^{2}\left\langle S y_{h}, y_{h}\right\rangle \leq\left\langle S_{h} y_{h}, y_{h}\right\rangle \leq\left\langle S y_{h}, y_{h}\right\rangle, \quad y_{h} \in Y_{h} .
$$

The following lemma shows that $K_{h}^{-1}$ inherits its continuity constant from the inf-sup constant.

Lemma 3.1. $\left\|K_{h}^{-1}\right\|_{\mathcal{L}\left(W^{\prime}, W\right)} \leq 2 \beta_{0}^{-2}-1$.

Proof. Define $\widetilde{K}_{h}\left(x_{h}, y_{h}\right)=\left(A_{h} x_{h}+B_{h} y_{h}, B_{h}^{\prime} x_{h}+2 S_{h} y_{h}\right)$ in $W_{h}=X_{h} \times Y_{h}$. As above, we find $\left\|K_{h}\right\|_{\mathcal{L}\left(W_{h}, W_{h}^{\prime}\right)}=$ $\left\|K_{h}^{-1}\right\|_{\mathcal{L}\left(W_{h}^{\prime}, W_{h}\right)}=1$ with respect to the discrete norm $\left\|\left(x_{h}, y_{h}\right)\right\|_{\widetilde{K}_{h}}^{2}=\left\langle\widetilde{K}_{h}\left(x_{h}, y_{h}\right),\left(x_{h}, y_{h}\right)\right\rangle$ in $W_{h}$. Next, we consider the natural embedding $E_{h} \in \mathcal{L}\left(W_{h}, W\right)$ with $E_{h}\left(x_{h}, y_{h}\right)=\left(x_{h}, y_{h}\right)$. From (3.2) and

$$
\begin{aligned}
\left\|\left(x_{h}, y_{h}\right)\right\|_{\widetilde{K}}^{2} & =\left\|x_{h}+A^{-1} B y_{h}\right\|_{A}^{2}+\left\langle S y_{h}, y_{h}\right\rangle=\left\|x_{h}+A_{h}^{-1} B_{h} y_{h}\right\|_{A}^{2}+\left\langle\left(2 S-S_{h}\right) y_{h}, y_{h}\right\rangle \\
& \leq\left\|x_{h}+A_{h}^{-1} B_{h} y_{h}\right\|_{A}^{2}+\left(2 \beta_{0}^{-2}-1\right)\left\langle S_{h} y_{h}, y_{h}\right\rangle \leq\left(2 \beta_{0}^{-2}-1\right)\left\|\left(x_{h}, y_{h}\right)\right\|_{\widetilde{K}_{h}}^{2}
\end{aligned}
$$

we obtain the bound $\left\|E_{h}\right\|_{\mathcal{L}\left(W_{h}, W\right)} \leq \sqrt{2 \beta_{0}^{-2}-1}$, and the assertion follows from

$$
\left\|K_{h}^{-1}\right\|_{\mathcal{L}\left(W^{\prime}, W\right)} \leq\left\|E_{h}\right\|_{\mathcal{L}\left(W_{h}, W\right)}\left\|K_{h}^{-1}\right\|_{\mathcal{L}\left(W_{h}^{\prime}, W_{h}\right)}\left\|E_{h}^{\prime}\right\|_{\mathcal{L}\left(W^{\prime}, W_{h}^{\prime}\right)}=\left\|E_{h}\right\|_{\mathcal{L}\left(W_{h}, W\right)}^{2} \leq 2 \beta_{0}^{-2}-1 .
$$

For given $\left(x^{\mathrm{sol}}, y^{\mathrm{sol}}\right) \in W$, let $\left(x_{h}^{\mathrm{sol}}, y_{h}^{\mathrm{sol}}\right) \in W_{h}$ be the Galerkin approximation, i.e., $\left(x_{h}^{\mathrm{sol}}, y_{h}^{\mathrm{sol}}\right) \in W_{h}$ is the unique solution of

$$
\left\langle K_{h}\left(x_{h}^{\mathrm{sol}}, y_{h}^{\mathrm{sol}}\right),\left(x_{h}, y_{h}\right)\right\rangle=\left\langle K\left(x^{\mathrm{sol}}, y^{\mathrm{sol}}\right),\left(x_{h}, y_{h}\right)\right\rangle, \quad\left(x_{h}, y_{h}\right) \in W_{h} .
$$

Then we obtain the following upper bound for the discretization error in terms of the best approximation error.

Theorem 3.2. $\left\|\left(x^{\mathrm{sol}}, y^{\mathrm{sol}}\right)-\left(x_{h}^{\mathrm{sol}}, y_{h}^{\mathrm{sol}}\right)\right\|_{\widetilde{K}} \leq\left(2 \beta_{0}^{-2}-1\right) \inf _{w_{h} \in W_{h}}\left\|\left(x^{\mathrm{sol}}, y^{\mathrm{sol}}\right)-w_{h}\right\|_{\widetilde{K}}$.

Proof. We use the observation in [33] on the Hilbert space norm of idempotents. The Galerkin property yields $\left(K_{h}^{-1} K\right)\left(K_{h}^{-1} K\right)=K_{h}^{-1} K_{h} K_{h}^{-1} K=K_{h}^{-1} K$, so that $K_{h}^{-1} K$ is non-trivial and idempotent. Then, Kato's identity, see, e.g., [31], reads $\left\|\mathrm{id}_{W}-K_{h}^{-1} K\right\|_{\mathcal{L}(W, W)}=\left\|K_{h}^{-1} K\right\|_{\mathcal{L}(W, W)}$. In terms of Lemma 3.1, the Galerkin orthogonality for all $\left(x_{h}, y_{h}\right) \in W_{h}$ gives

$$
\begin{aligned}
\left\|\left(x^{\mathrm{sol}}, y^{\mathrm{sol}}\right)-\left(x_{h}^{\mathrm{sol}}, y_{h}^{\mathrm{sol}}\right)\right\|_{\widetilde{K}} & =\left\|\left(\mathrm{id}_{W}-K_{h}^{-1} K\right)\left(x^{\mathrm{sol}}, y^{\mathrm{sol}}\right)\right\|_{\widetilde{K}} \\
& =\left\|\left(\mathrm{id}_{W}-K_{h}^{-1} K\right)\left(\left(x^{\mathrm{sol}}, y^{\mathrm{sol}}\right)-\left(x_{h}, y_{h}\right)\right)\right\|_{\widetilde{K}} \\
& \leq\|K\|_{\mathcal{L}\left(W, W^{\prime}\right)}\left\|K_{h}^{-1}\right\|_{\mathcal{L}\left(W^{\prime}, W\right)}\left\|\left(x^{\mathrm{sol}}, y^{\mathrm{sol}}\right)-\left(x_{h}, y_{h}\right)\right\|_{\widetilde{K}} \\
& \leq\left(2 \beta_{0}^{-2}-1\right)\left\|\left(x^{\mathrm{sol}}, y^{\mathrm{sol}}\right)-\left(x_{h}, y_{h}\right)\right\|_{\widetilde{K}} .
\end{aligned}
$$


As we have already seen for the model problem, a well-posed Petrov-Galerkin formulation can be related to a saddle point formulation. Here we state the reverse. The saddle point approach also provides a Petrov-Galerkin discretization. Therefore, we consider a right-hand side $f \in B(Y) \subset X^{\prime}$, and let $y^{\text {sol }}=S^{-1} B^{\prime} A^{-1} f \in Y$ be the unique solution of the equation $B y^{\text {sol }}=f$. Then, we observe that the approximation $y_{h}^{\text {sol }}=S_{h}^{-1} B_{h}^{\prime} A_{h}^{-1} f$ is the unique Petrov-Galerkin solution of the variational problem: find $y_{h}^{\text {sol }} \in Y_{h}$ such that

$$
\left\langle B_{h} y_{h}^{\text {sol }}, x_{h}\right\rangle=\left\langle f, x_{h}\right\rangle, \quad x_{h} \in X_{h}^{\mathrm{red}}:=A_{h}^{-1} B_{h}\left(Y_{h}\right) \subset X_{h} .
$$

The operator $S_{h}^{-1} B_{h}^{\prime} A_{h}^{-1} B$ is idempotent, and we have

$$
\|B\|_{\mathcal{L}\left(Y, X^{\prime}\right)}=\left\|A_{h}^{-1}\right\|_{\mathcal{L}\left(X^{\prime}, X_{h}\right)}=1, \quad\left\|B_{h}^{\prime}\right\|_{\mathcal{L}\left(X_{h}, Y^{\prime}\right)}=\left\|S_{h}^{-1}\right\|_{\mathcal{L}\left(Y^{\prime}, Y\right)} \leq \beta_{0}^{-1} .
$$

Following the lines of the proof of Theorem 3.2 (see also [33], Thm. 2.1), we obtain

$$
\left\|y^{\mathrm{sol}}-y_{h}^{\mathrm{sol}}\right\|_{S} \leq \beta_{0}^{-1} \inf _{y_{h} \in Y_{h}}\left\|y^{\mathrm{sol}}-y_{h}\right\|_{S}
$$

provided that we can show that $\left\|S_{h}^{-1} B_{h}^{\prime}\right\|_{\mathcal{L}\left(X_{h}, Y\right)} \leq \beta_{0}^{-1}$. To do so, we start with the straightforward estimate

$$
\begin{aligned}
\left\|S_{h}^{-1} B_{h}^{\prime} x_{h}\right\|_{S_{h}}^{2} & =\left\langle x_{h}, B_{h} S_{h}^{-1} B_{h}^{\prime} x_{h}\right\rangle \leq\left\|x_{h}\right\|_{A}\left\langle A_{h}^{-1} B_{h} S_{h}^{-1} B_{h}^{\prime} x_{h}, B_{h} S_{h}^{-1} B_{h}^{\prime} x_{h}\right\rangle^{\frac{1}{2}} \\
& =\left\|x_{h}\right\|_{A}\left\langle x_{h}, B_{h} S_{h}^{-1} B_{h}^{\prime} A_{h}^{-1} B_{h} S_{h}^{-1} B_{h}^{\prime} x_{h}\right\rangle^{\frac{1}{2}}=\left\|x_{h}\right\|_{A}\left\langle x_{h}, B_{h} S_{h}^{-1} B_{h}^{\prime} x_{h}\right\rangle^{\frac{1}{2}}=\left\|x_{h}\right\|_{A}\left\|S_{h}^{-1} B_{h}^{\prime} x_{h}\right\|_{S_{h}} .
\end{aligned}
$$

Using now the upper spectral bound for $S$, we find $\left\|S_{h}^{-1} B_{h}^{\prime} x_{h}\right\|_{S} \leq \beta_{0}^{-1}\left\|S_{h}^{-1} B_{h}^{\prime} x_{h}\right\|_{S_{h}} \leq \beta_{0}^{-1}\left\|x_{h}\right\|_{A}$.

Remark 3.3. Additionally, the identity

$$
\sup _{x_{h} \in X_{h}^{\text {red }}} \frac{\left|\left\langle B_{h} y_{h}, x_{h}\right\rangle\right|^{2}}{\left\|x_{h}\right\|_{A}^{2}}=\sup _{\tilde{y}_{h} \in Y_{h}} \frac{\left|\left\langle B_{h} y_{h}, A_{h}^{-1} B_{h} \tilde{y}_{h}\right\rangle\right|^{2}}{\left\|A_{h}^{-1} B_{h} \tilde{y}_{h}\right\|_{A}^{2}}=\sup _{\tilde{y}_{h} \in Y_{h}} \frac{\left\langle S_{h} y_{h}, \tilde{y}_{h}\right\rangle^{2}}{\left\langle S_{h} \tilde{y}_{h}, \tilde{y}_{h}\right\rangle}=\left\|y_{h}\right\|_{S_{h}}^{2}
$$

implies $\inf _{y_{h} \in Y_{h}} \sup _{x_{h} \in X_{h}^{\text {red }}} \frac{\left|\left\langle y_{h}, B_{h}^{\prime} x_{h}\right\rangle\right|}{\left\|x_{h}\right\|_{A}\left\|y_{h}\right\|_{S}} \geq \beta_{0}$ for the reduced space $X_{h}^{\text {red }} \subset X_{h}$ (see also [20], Thm. 2.1).

Before we focus on stability estimates in the next section, we relate the Schur complement norm (3.1) with an inf-sup constant. We assume that $\gamma>0$ exists such that

$$
\sup _{y \in Y} \frac{|\langle B y, x\rangle|}{\|y\|_{Y}} \geq \gamma\|x\|_{X}, \quad x \in X .
$$

Then, since $B$ is injective, we obtain by duality $\|y\|_{S}=\sup _{x \in X} \frac{|\langle B y, x\rangle|}{\|x\|_{A}} \geq \gamma\|y\|_{Y}$ for $y \in Y$ (cf. [6], Lem. 4.4.2).

\section{Stability ESTimates FOR FIRST-ORDER SyStems}

For representative first-order differential operators $L$ we specify the stability constant $C_{L}<\infty$.

\subsection{An abstract framework}

Let $H$ be a Hilbert space with norm $\|\cdot\|_{H}$, and let $U \subset H$ be a dense subspace. We assume that $L: U \longrightarrow H$ is a closed linear operator, i.e., the graph of the operator $\{(u, L u): u \in U\}$ is closed in $H \times H$, the domain $U$ is a Hilbert space in the graph norm $\|u\|_{U}=\sqrt{\|u\|_{H}^{2}+\|L u\|_{H}^{2}}$, and the operator $L$ has closed range. Moreover we assume that $L$ is injective and surjective, and that its inverse is bounded.

In our examples, this setting is obtained as follows: for a dense subset $\mathcal{D}(L) \subset H$, we verify the existence of a stability constant $C_{L}<\infty$ satisfying

$$
\|u\|_{H} \leq C_{L}\|L u\|_{H}, \quad u \in \mathcal{D}(L) .
$$

We then define $U$ as the closure of $\mathcal{D}(L)$ with respect to the graph norm $\|u\|_{U}^{2}=\|u\|_{H}^{2}+\|L u\|_{H}^{2}$ and $L$ can be extended to $U$. The stability estimate (4.1) holds also for $u \in U$, and the range $L(U) \subset H$ is closed. Thus the choice $H=L(U)$ guarantees that we are in the above abstract setting. 


\subsection{Examples of second order partial differential equations and first order systems}

We recall that $d=2,3$ is the spatial dimension and that $\Omega \subset \mathbb{R}^{d}$ is a bounded Lipschitz domain. Let $\partial \Omega=\partial \Omega_{\mathrm{D}} \cup \partial \Omega_{\mathrm{N}}$ be a decomposition of the boundary into Dirichlet and Neumann part, and let $n$ be the outer unit normal on $\partial \Omega$. For simplicity, we consider, without loss of generality, only homogeneous boundary conditions. The standard $\mathrm{L}_{2}$-norm on $\Omega$ is denoted by $\|\cdot\|_{\Omega}$. In our first examples, we start with a second order partial differential equation and transform it to a first-order system. In all cases, the Hilbert space $H$ is a subset of $\mathrm{L}_{2}\left(\Omega, \mathbb{R}^{M}\right)$.

A scalar elliptic model problem. We reconsider the model problem (2.1) with Dirichlet boundary $\partial \Omega_{\mathrm{D}}=\partial \Omega$ and $\mathcal{D}(L)=\mathrm{H}(\operatorname{div}, \Omega) \times \mathrm{H}_{0}^{1}(\Omega)$. Let $C_{\mathrm{P}}>0$ be the Poincaré constant with $\|p\|_{\Omega} \leq C_{\mathrm{P}}\|\nabla p\|_{\Omega}$ for $p \in \mathrm{H}_{0}^{1}(\Omega)$. Furthermore, the continuous Sobolev embedding $\mathrm{H}^{1}(\Omega) \subset \mathrm{L}_{4}(\Omega)$ yields that there exists a $C_{b}>0$ such that

$$
\|b p\|_{\Omega} \leq\|p\|_{\mathrm{L}_{4}(\Omega)}\|b\|_{\mathrm{L}_{4}(\Omega)} \leq C_{b}\|\nabla p\|_{\Omega}, \quad p \in \mathrm{H}_{0}^{1}(\Omega) .
$$

The first-order operator $L$ is given in $(2.2)$.

Lemma 4.1. The stability constants of the scalar elliptic model problem can be defined as

$$
C_{L}=\sqrt{2+\left(4+C_{P}^{2}+4 C_{b}^{2}\right)\left(1+C_{\mathrm{P}}^{2}\right)}
$$

Proof. To verify (4.1), we firstly provide an upper bound of $\|\nabla p\|_{\Omega}$ in terms of $\|L(\sigma, p)\|_{\Omega}$. Due to the homogeneous Dirichlet boundary condition and the assumption $\nabla \cdot b=0$ on $b$, we have $(p, b \cdot \nabla p)_{\Omega}=$ $-(p, b \cdot \nabla p)_{\Omega}+\left(p^{2}, b \cdot n\right)_{\partial \Omega}=-(p, b \cdot \nabla p)_{\Omega}$, which gives $(p, b \cdot \nabla p)_{\Omega}=0$. Then, we get

$$
\begin{aligned}
\|\nabla p\|_{\Omega}^{2} \leq\|\nabla p\|_{\Omega}^{2}+(a p, p)_{\Omega} & =\|\nabla p\|_{\Omega}^{2}-(p, b \cdot \nabla p)_{\Omega}+(a p, p)_{\Omega} \\
& =(\sigma-b p+\nabla p, \nabla p)_{\Omega}+(\nabla \cdot \sigma, p)_{\Omega}+(a p, p)_{\Omega}=(L(\sigma, p),(\nabla p, p))_{\Omega} \\
& \leq\|L(\sigma, p)\|_{\Omega}\|(\nabla p, p)\|_{\Omega} \leq \sqrt{1+C_{\mathrm{P}}^{2}}\|L(\sigma, p)\|_{\Omega}\|\nabla p\|_{\Omega} .
\end{aligned}
$$

Secondly, we bound $\|\sigma\|_{\Omega}$ in terms of $\|L(\sigma, p)\|_{\Omega}$ and $\|\nabla p\|_{\Omega}$ by

$$
\|\sigma\|_{\Omega}^{2}=(\sigma-b p+\nabla p, \sigma)_{\Omega}+(b p-\nabla p, \sigma)_{\Omega} \leq\left(\|L(\sigma, p)\|_{\Omega}+\|b p-\nabla p\|_{\Omega}\right)\|\sigma\|_{\Omega}
$$

which yields $\|\sigma\|_{\Omega} \leq\|L(\sigma, p)\|_{\Omega}+\|b p-\nabla p\|_{\Omega}$. This gives the estimate

$$
\begin{aligned}
\|\sigma\|_{\Omega}^{2}+\|p\|_{\Omega}^{2} & \leq 2\|L(\sigma, p)\|_{\Omega}^{2}+2\|b p-\nabla p\|_{\Omega}^{2}+\|p\|_{\Omega}^{2}=2\|L(\sigma, p)\|_{\Omega}^{2}+4\|b p\|^{2}+4\|\nabla p\|_{\Omega}^{2}+\|p\|_{\Omega}^{2} \\
& \leq 2\|L(\sigma, p)\|_{\Omega}^{2}+\left(4 C_{b}^{2}+4+C_{\mathrm{P}}^{2}\right)\|\nabla p\|_{\Omega}^{2} \leq\left(2+\left(4 C_{b}^{2}+4+C_{\mathrm{P}}^{2}\right)\left(1+C_{\mathrm{P}}^{2}\right)\right)\|L(\sigma, p)\|_{\Omega}^{2} .
\end{aligned}
$$

We note that the stability constant does not depend on the norm of $a$ but depends on $b$. Stable estimates for dominating convection are considered in $[12,18]$ with suitable weighted norms in order to compensate strong boundary layers. Stability for the pure transport problem is discussed in Theorem 2.4 from [13].

The Helmholtz problem. As second example, we consider the Helmholtz equation

$$
-\Delta p-\omega^{2} p=\mathrm{i} \omega g \quad \text { in } \Omega, \quad \nabla p \cdot n+i \omega p=0 \quad \text { on } \partial \Omega,
$$

where $\omega>0$ is a given positive wave number. Introducing $\sigma=-1 /(\mathrm{i} \omega) \nabla p$, we obtain $L(\sigma, p)=(0, g)$ with

$$
L(\sigma, p)=(\mathrm{i} \omega \sigma+\nabla p, \mathrm{i} \omega p+\nabla \cdot \sigma)
$$

defined on $\mathcal{D}(L)=\left\{(\sigma, p) \in \mathrm{H}(\operatorname{div}, \Omega) \times \mathrm{H}^{1}(\Omega): \sigma \cdot n-p=0\right.$ on $\left.\partial \Omega\right\} \subset H$ with $H=\mathrm{L}_{2}\left(\Omega, \mathbb{C}^{d} \times \mathbb{C}\right)$. 
Lemma 4.2. Assume that a constant $\alpha>0$ exists such that $x \cdot n(x) \geq \alpha$ for a.a. $x \in \partial \Omega$. Then, the stability constant for the Helmholtz problem can be defined as

$$
C_{L}=3 R+\frac{R^{2}}{\alpha}+\frac{d-1}{\omega^{2}}
$$

where $d$ is the space dimension and $|x|<R$ for $x \in \partial \Omega$.

We note that such a constant $\alpha$ exists, e.g., for star-shaped domains with the origin placed in $\Omega$.

Proof. Following the ideas in [28] Proposition 8.1.4, [16,19], Section 4.3, the stability estimate can be shown in three steps. Firstly, we provide upper bounds for $\|p\|_{\partial \Omega}$ and $\|\sigma\|_{\Omega}$. Secondly we consider smooth curl-free vector fields $\sigma$ and bound $\|p\|_{\Omega}$. Finally a Helmholtz decomposition is applied.

Using the boundary conditions for $(\sigma, p) \in \mathcal{D}(L)$, we get $\|p\|_{\partial \Omega}^{2}=(\sigma \cdot n, p)_{\partial \Omega}=(\nabla p, \sigma)_{\Omega}+(p, \nabla \cdot \sigma)_{\Omega}$. The definition of $L(\sigma, p)$ now yields

$$
\begin{aligned}
\|p\|_{\partial \Omega}^{2} & =\operatorname{Re}\left(\mathrm{i} \omega\|(\sigma, p)\|_{\Omega}^{2}+(\nabla p, \sigma)_{\Omega}+(\nabla \cdot \sigma, p)_{\Omega}\right) \\
& =\operatorname{Re}\left((\mathrm{i} \omega \sigma+\nabla p, \sigma)_{\Omega}+(\mathrm{i} \omega p+\nabla \cdot \sigma, p)_{\Omega}\right)=\operatorname{Re}(L(\sigma, p),(\sigma, p))_{\Omega} \leq\|L(\sigma, p)\|_{\Omega}\|(\sigma, p)\|_{\Omega} \\
\|\sigma\|_{\Omega}^{2} & =\|p\|_{\Omega}^{2}+\frac{1}{\omega^{2}} \operatorname{Re}(L(\sigma, p),(\mathrm{i} \omega \sigma,-\mathrm{i} \omega p))_{\Omega} \leq\|p\|_{\Omega}^{2}+\frac{1}{\omega^{2}}\|L(\sigma, p)\|_{\Omega}\|(\sigma, p)\|_{\Omega} .
\end{aligned}
$$

In the second step, we restrict ourselves to $(\sigma, p) \in \mathcal{D}(L)$ such that $\sigma$ is smooth enough and $\nabla \times \sigma=0$. A crucial role within this step plays the following equalities which can be easily established by a straightforward computation

$$
\begin{aligned}
& \nabla \cdot\left(|\sigma|^{2} x\right)=\sigma \cdot \overline{\nabla(\sigma \cdot x)}+\bar{\sigma} \cdot \nabla(\sigma \cdot x)+(d-2)|\sigma|^{2}, \\
& \nabla \cdot\left(|p|^{2} x\right)=p \overline{\nabla p \cdot x}+\nabla p \cdot x \bar{p}+d|p|^{2} .
\end{aligned}
$$

In terms of (4.6), we can now express boundary terms by volume terms and find by integration by parts

$$
\begin{aligned}
& \left(|\sigma|^{2}, x \cdot n\right)_{\partial \Omega}=2 \operatorname{Re}(\sigma, \nabla(x \cdot \sigma))_{\Omega}+(d-2)(\sigma, \sigma)_{\Omega}, \\
& \left(|p|^{2}, x \cdot n\right)_{\partial \Omega}=2 \operatorname{Re}(p, x \cdot \nabla p)_{\Omega}+d(p, p)_{\Omega} .
\end{aligned}
$$

A reformulation of $\operatorname{Re}(L(\sigma, p),(x p, x \cdot \sigma))_{\Omega}$ in terms of (4.7) and the use of the boundary condition give

$$
\begin{aligned}
2 \operatorname{Re}(L(\sigma, p),(x p, x \cdot \sigma))_{\Omega} & =2 \operatorname{Re}(\nabla \cdot \sigma, x \cdot \sigma)_{\Omega}+2 \operatorname{Re}(p, x \cdot \nabla p)_{\Omega} \\
& =2 \operatorname{Re}(n \cdot \sigma, x \cdot \sigma)_{\partial \Omega}-2 \operatorname{Re}(\sigma, \nabla(x \cdot \sigma))_{\Omega}+2 \operatorname{Re}(p, x \cdot \nabla p)_{\Omega} \\
& =2 \operatorname{Re}(p, x \cdot \sigma)_{\partial \Omega}-\left(|\sigma|^{2}, x \cdot n\right)_{\partial \Omega}+(d-2)(\sigma, \sigma)_{\Omega}+\left(|p|^{2}, x \cdot n\right)_{\partial \Omega}-d(p, p)_{\Omega}
\end{aligned}
$$

or equivalently

$$
d\|p\|_{\Omega}^{2}+\left(|\sigma|^{2}, x \cdot n\right)_{\partial \Omega}=\left(|p|^{2}, x \cdot n\right)_{\partial \Omega}+2 \operatorname{Re}(p, x \cdot \sigma)_{\partial \Omega}+(d-2)(\sigma, \sigma)_{\Omega}-2 \operatorname{Re}(L(\sigma, p),(x p, x \cdot \sigma))_{\Omega} .
$$

Using the assumptions on $\Omega$ there exits $R<\infty$ with $|x|<R$ for $x \in \partial \Omega$ and thus $\alpha \leq x \cdot n \leq R$ for almost all $x \in \partial \Omega$. This observation guarantees that we can provide an upper bound for $\|p\|_{\Omega}^{2}$ by using Young's inequality

$$
\begin{gathered}
d\|p\|_{\Omega}^{2}+\alpha\|\sigma\|_{\partial \Omega}^{2} \leq R\|p\|_{\partial \Omega}^{2}+2 R\|p\|_{\partial \Omega}\|\sigma\|_{\partial \Omega}+(d-2)\|\sigma\|_{\Omega}^{2}+2 R\|L(\sigma, p)\|_{\Omega}\|(\sigma, p)\|_{\Omega}, \\
d\|p\|_{\Omega}^{2} \leq\left(R+\frac{R^{2}}{\alpha}\right)\|p\|_{\partial \Omega}^{2}+2 R\|L(\sigma, p)\|_{\Omega}\|(\sigma, p)\|_{\Omega}+(d-2)\|\sigma\|_{\Omega}^{2} .
\end{gathered}
$$


Now the upper bounds (4.5a) and (4.5b) can be used, and we get

$$
\begin{aligned}
d\|p\|_{\Omega}^{2} & \leq\left(R+\frac{R^{2}}{\alpha}+2 R+\frac{d-2}{\omega^{2}}\right)\|L(\sigma, p)\|_{\Omega}\|(\sigma, p)\|_{\Omega}+(d-2)\|p\|_{\Omega}^{2}, \\
\|p\|_{\Omega}^{2} & \leq \frac{1}{2}\left(3 R+\frac{R^{2}}{\alpha}+\frac{d-2}{\omega^{2}}\right)\|L(\sigma, p)\|_{\Omega}\|(\sigma, p)\|_{\Omega} .
\end{aligned}
$$

Combining the results of the first two steps, we obtain for all $(\sigma, p) \in \mathcal{D}(L)$ with $\sigma=\nabla \psi$ the stability bound

$$
\|(\sigma, p)\|_{\Omega} \leq\left(3 R+\frac{R^{2}}{\alpha}+\frac{d-1}{\omega^{2}}\right)\|L(\sigma, p)\|_{\Omega}=C_{L}\|L(\sigma, p)\|_{\Omega} .
$$

A density argument yields that the above estimate hold also for all $(\sigma, p) \in \mathcal{D}(L)$ with $\nabla \times \sigma=0$.

Finally in the third step, we have to show that the above stability estimate holds true for all $(\sigma, p) \in \mathcal{D}(L)$. To do so, we use a Helmholtz decomposition. Introducing

$$
H_{0}=\left\{\sigma \in \mathrm{L}_{2}\left(\Omega, \mathbb{C}^{d}\right):(\sigma, \nabla \times v)_{\Omega}=0 \text { for all } v \in \mathrm{H}_{0}(\operatorname{curl}, \Omega)\right\},
$$

$(\sigma, p) \in \mathcal{D}(L)$ has a unique orthogonal decomposition into $\left(\sigma_{0}, p\right)+\left(\sigma_{1}, 0\right)$ with $\sigma_{0} \in H_{0}$ and $\left(\sigma_{1}, \nabla q\right)_{\Omega}=0$ for all $q \in H^{1}(\Omega)$. Then the definition of $L$ trivially gives $L\left(\sigma_{1}, 0\right)=\left(\mathrm{i} \omega \sigma_{1}, 0\right)$. Moreover the operator $L$ preserves the orthogonality between $\left(\sigma_{0}, p\right)$ and $\left(\sigma_{1}, 0\right)$. These observations guarantee now in terms of $(4.8)$

$$
\begin{aligned}
\|(\sigma, p)\|_{\Omega}^{2}=\left\|\left(\sigma_{0}, p\right)\right\|_{\Omega}^{2}+\left\|\left(\sigma_{1}, 0\right)\right\|_{\Omega}^{2} & \leq C_{L}^{2}\left\|L\left(\sigma_{0}, p\right)\right\|_{\Omega}^{2}+\frac{1}{\omega^{2}}\left\|L\left(\sigma_{1}, p\right)\right\|_{\Omega}^{2} \\
& \leq C_{L}^{2}\left(\left\|L\left(\sigma_{0}, p\right)\right\|_{\Omega}^{2}+\left\|L\left(\sigma_{1}, p\right)\right\|_{\Omega}^{2}\right)=C_{L}^{2}\|L(\sigma, p)\|_{\Omega}^{2} .
\end{aligned}
$$

Remark 4.3. We note that the stability constant $C_{L}$ is uniformly bounded for large wave numbers.

Linear elasticity. We consider the linear elasticity system

$$
-\operatorname{div}(\mathcal{C} \varepsilon(u))=f \quad \text { in } \Omega, \quad u=0 \text { on } \partial \Omega_{\mathrm{D}} \text { and } \mathcal{C} \varepsilon(u) n=0 \text { on } \partial \Omega_{\mathrm{N}},
$$

where $\mathcal{C}$ is the positive definite elasticity tensor in $\mathbb{R}_{\mathrm{sym}}^{d \times d}, \varepsilon(u)=\operatorname{sym}(\mathrm{D} u)$ is the linearized strain tensor, $f \in \mathrm{L}_{2}\left(\Omega, \mathbb{R}^{d}\right)$ is a prescribed volume load, and $u$ is the displacement. We assume that $\partial \Omega_{\mathrm{D}}$ has a positive measure in $d-1$. Introducing the stress $\sigma=\mathcal{C} \varepsilon(u)$, we can rewrite (4.9) as a first-order system $L(\sigma, u)=(0,-f)$ with

$$
L(\sigma, u)=(\sigma-\mathcal{C} \varepsilon(u), \operatorname{div}(\sigma))
$$

and domain $\mathcal{D}(L) \subset H=\mathrm{L}_{2}\left(\Omega, \mathbb{R}_{\mathrm{sym}}^{d \times d} \times \mathbb{R}^{d}\right)$ given by

$$
\mathcal{D}(L)=\left\{(\sigma, u) \in \mathrm{H}(\operatorname{div}, \Omega)^{d} \times \mathrm{H}^{1}(\Omega)^{d}: \sigma^{\top}=\sigma, \sigma n=0 \text { on } \partial \Omega_{\mathrm{N}} \text { and } u=0 \text { on } \partial \Omega_{\mathrm{D}}\right\} .
$$

On $H$ we use the norm $\|(\sigma, u)\|_{H}^{2}=\left(\mathcal{C}^{-1} \sigma, \sigma\right)_{\Omega}+\|u\|_{\Omega}^{2}$ and recall that the stability constant has to be specified with respect to this norm.

Lemma 4.4. The stability constant of the elasticity problem can be defined as

$$
C_{L}=\sqrt{1+2 C_{K}^{2}\left|\mathcal{C}^{-1}\right|\left(1+C_{K}^{2}\left|\mathcal{C}^{-1}\right|\right)}
$$


Proof. Firstly we provide a bound for $\|u\|_{\Omega}$ by $\|L(\sigma, u)\|_{H}$. By assumption the Dirichlet boundary part is nontrivial, and thus no rigid body motion is in $\mathcal{D}(L)$ and Korn's inequality shows, i.e., for all $u \in \mathrm{H}^{1}(\Omega)^{d}$ with $u=0$ on $\partial \Omega_{\mathrm{D}}$ there exists a constant $C_{\mathrm{K}}>0$ only depending on $\Omega$ and $\partial \Omega_{\mathrm{D}}$ such that

$$
\|u\|_{\Omega}^{2} \leq C_{\mathrm{K}}^{2}\|\varepsilon(u)\|_{\Omega}^{2} \leq C_{\mathrm{K}}^{2}\left|\mathcal{C}^{-1}\right|(\mathcal{C} \varepsilon(u), \varepsilon(u))_{\Omega} .
$$

Korn's inequality show that to bound $\|u\|_{\Omega}^{2}$, we have to bound $(\mathcal{C} \varepsilon(u), \varepsilon(u))_{\Omega}$. For $(\sigma, u) \in \mathcal{D}(L)$ we have, due to the boundary conditions,

$$
\begin{aligned}
(\mathcal{C} \varepsilon(u), \varepsilon(u))_{\Omega} & =-(\sigma-\mathcal{C} \varepsilon(u), \varepsilon(u))_{\Omega}+(\sigma, \varepsilon(u))_{\Omega}=-(\sigma-\mathcal{C} \varepsilon(u), \varepsilon(u))_{\Omega}-(\operatorname{div}(\sigma), u)_{\Omega} \\
& \leq\|L(\sigma, u)\|_{H}\|(\mathcal{C} \varepsilon(u), u)\|_{H} \leq\|L(\sigma, u)\|_{H}\left(1+C_{\mathrm{K}}^{2}\left|\mathcal{C}^{-1}\right|\right)^{\frac{1}{2}}(\mathcal{C} \varepsilon(u), \varepsilon(u))_{\Omega}^{\frac{1}{2}}
\end{aligned}
$$

and thus $\|u\|_{\Omega}^{2} \leq C_{\mathrm{K}}^{2}\left|\mathcal{C}^{-1}\right|\left(1+C_{\mathrm{K}}^{2}\left|\mathcal{C}^{-1}\right|\right)\|L(\sigma, u)\|_{H}^{2}$.

Secondly we bound $\left(\mathcal{C}^{-1} \sigma, \sigma\right)_{\Omega}$ by $\|L(\sigma, u)\|_{H}$ and $\|u\|_{\Omega}$. Integration by parts and the homogeneous boundary conditions guarantee

$$
\begin{aligned}
\left(\mathcal{C}^{-1} \sigma, \sigma\right)_{\Omega} & =\left(\sigma-\mathcal{C} \varepsilon(u), \mathcal{C}^{-1} \sigma\right)_{\Omega}+(\varepsilon(u), \sigma)_{\Omega}=\left(\sigma-\mathcal{C} \varepsilon(u), \mathcal{C}^{-1} \sigma\right)_{\Omega}-(\operatorname{div}(\sigma), u)_{\Omega} \\
& \leq\|L(\sigma, u)\|_{H}\|(\sigma, u)\|_{H} \leq \frac{1}{2}\|L(\sigma, u)\|_{H}+\frac{1}{2}\left(\mathcal{C}^{-1} \sigma, \sigma\right)_{\Omega}+\frac{1}{2}\|u\|_{\Omega}^{2} .
\end{aligned}
$$

Combining these two bounds, we obtain the assertion by

$$
\|(\sigma, u)\|_{H}^{2} \leq\|L(\sigma, u)\|_{H}^{2}+2\|u\|_{\Omega}^{2} \leq\left(1+2 C_{\mathrm{K}}^{2}\left|\mathcal{C}^{-1}\right|\left(1+C_{\mathrm{K}}^{2}\left|\mathcal{C}^{-1}\right|\right)\right)\|L(\sigma, u)\|_{H}^{2} .
$$

Remark 4.5. Since $\left|\mathcal{C}^{-1}\right|$ is bounded in the incompressible limit, the estimate is robust for nearly incompressible materials.

The Stokes system. We consider the stationary linear Stokes system which can be regarded as the incompressible limit of the elasticity system

$$
\begin{aligned}
-\Delta u+\nabla p & =f, \quad \text { in } \Omega, \quad u=0 \text { on } \partial \Omega . \\
\operatorname{div}(u) & =0
\end{aligned}
$$

Introducing $\sigma$ by $\sigma=\varepsilon(u)-p I$, we can rewrite (4.11) as a first-order system $L(\sigma, u, p)=(0,-f, 0)$ with

$$
L(\sigma, u, p)=(\sigma+p I-\varepsilon(u), \operatorname{div}(\sigma), \operatorname{div}(u))
$$

defined on $\mathcal{D}(L)=\left\{(\sigma, u, p) \in \mathrm{H}(\operatorname{div}, \Omega)^{d} \times \mathrm{H}^{1}(\Omega)^{d} \times \mathrm{L}_{2}(\Omega): \sigma^{\top}=\sigma, u=0\right.$ on $\left.\partial \Omega, \int_{\Omega} p \mathrm{~d} x=0\right\}$.

Lemma 4.6. The stability constant of the Stokes problem can be defined as

$$
C_{L}=\sqrt{C_{1}^{2}\left(C_{K}^{2}+\sqrt{3} / c_{p}^{2}\right)+\left(1+\left(\frac{\sqrt{3 d}}{c_{p}}+1\right) C_{1}\right)^{2}}
$$

with $C_{1}=\sqrt{1+d+C_{K}^{2}}$ and the inf-sup constant $c_{p}$.

Proof. We proceed in three steps and consider the terms $\|\varepsilon(u)\|_{\Omega},\|p\|_{\Omega}$ and $\|\sigma\|_{\Omega}$ separately. For $(\sigma, u, p) \in \mathcal{D}(L)$ Korn's inequality can be applied and thus, we get

$$
\begin{aligned}
\|\varepsilon(u)\|_{\Omega}^{2} & =(\varepsilon(u)-\sigma-p I, \varepsilon(u))_{\Omega}+(\sigma, \varepsilon(u))_{\Omega}+(p I, \varepsilon(u))_{\Omega} \\
& =(\varepsilon(u)-\sigma-p I, \varepsilon(u))_{\Omega}-(\operatorname{div}(\sigma), u)_{\Omega}+(p, \operatorname{div}(u))_{\Omega} \\
& \leq\|L(\sigma, u, p)\|_{\Omega}\|(\varepsilon(u), u, \operatorname{div}(u))\|_{\Omega} \leq \sqrt{1+C_{\mathrm{K}}^{2}+d}\|L(\sigma, u, p)\|_{\Omega}\|\varepsilon(u)\|_{\Omega} .
\end{aligned}
$$


To bound $\|p\|_{\Omega}$, we use an inf-sup estimate, which yields

$$
\begin{aligned}
c_{p}\|p\|_{\Omega} & \leq \sup _{(0, v, 0) \in \mathcal{D}(L)} \frac{(p, \operatorname{div}(v))_{\Omega}}{\|\varepsilon(v)\|_{\Omega}} \leq \sup _{(0, v, 0) \in \mathcal{D}(L)} \frac{(p I+\sigma, \varepsilon(v))_{\Omega}+(\operatorname{div}(\sigma), v)_{\Omega}}{\|\varepsilon(v)\|_{\Omega}} \\
& \leq\|p I+\sigma-\varepsilon(u)\|_{\Omega}+\|\varepsilon(u)\|_{\Omega}+C_{\mathrm{K}}\|\operatorname{div}(\sigma)\|_{\Omega} \leq C_{1} \sqrt{3}\|L(\sigma, u, p)\|_{\Omega} .
\end{aligned}
$$

Finally in terms of the two previous upper estimates, we can bound

$$
\begin{aligned}
(\sigma, \sigma)_{\Omega} & =(\sigma-p I-\varepsilon(u), \sigma)_{\Omega}+(p I, \sigma)_{\Omega}+(\varepsilon(u), \sigma)_{\Omega} \\
& \leq\left(\|L(\sigma, u, p)\|_{\Omega}+\sqrt{d}\left\|_{p}\right\|_{\Omega}+\|\varepsilon(u)\|_{\Omega}\right)\|\sigma\|_{\Omega} \leq\left(1+\left(\frac{\sqrt{3 d}}{c_{p}}+1\right) C_{1}\right)\|L(\sigma, u, p)\|_{\Omega}\|\sigma\|_{\Omega}
\end{aligned}
$$

which yields $C_{L}^{2}=C_{1}^{2}\left(C_{\mathrm{K}}^{2}+\sqrt{3} / c_{p}^{2}\right)+\left(1+\left(\frac{\sqrt{3 d}}{c_{p}}+1\right) C_{1}\right)^{2}$.

Maxwell's equations. For linear materials, electro-magnetic waves are determined by the first-order system for the magnetic $\mathcal{H}$ and the electric $\mathcal{E}$ field

$$
L(\mathcal{H}, \mathcal{E})=\left(\partial_{t} \mathcal{H}+\mu^{-1} \nabla \times \mathcal{E}, \partial_{t} \mathcal{E}-\varepsilon^{-1} \nabla \times \mathcal{H}\right)
$$

Here the permeability $\mu$ and the permittivity $\varepsilon$ are positive constants. The boundary is disjointly decomposed in $\partial \Omega_{\mathcal{E}}$ and $\partial \Omega_{\mathcal{H}}$. In $\Omega$, we define

$$
\begin{array}{r}
\mathcal{D}(L)=\left\{\left(\mathcal{H}, \mathcal{E} \in \mathrm{L}_{2}\left((0, T), \mathrm{H}(\operatorname{curl}, \Omega)^{2}\right) \cap \mathrm{H}^{1}\left((0, T), \mathrm{L}_{2}(\Omega)^{6}\right): \operatorname{div}(\mu \mathcal{H})=\operatorname{div}(\varepsilon \mathcal{E})=0 \text { in } \Omega \times(0, T),\right.\right. \\
\left.\mathcal{H}(0)=\mathcal{E}(0)=0, \text { in } \Omega, \quad \mathcal{H} \times n=0 \text { on } \partial \Omega_{\mathcal{H}} \times(0, T), \mathcal{E} \times n=0 \text { on } \partial \Omega_{\mathcal{E}} \times(0, T)\right\} .
\end{array}
$$

By $H$ we denote the closure of $\mathcal{D}(L)$ in $\mathrm{L}_{2}\left((0, T) \times \Omega, \mathbb{R}^{3} \times \mathbb{R}^{3}\right)$ associated with the inner product

$$
((\mathcal{H}, \mathcal{E}),(\tilde{\mathcal{H}}, \tilde{\mathcal{E}}))_{H}=\int_{0}^{T}\left((\mu \mathcal{H}(t), \tilde{\mathcal{H}}(t))_{\Omega}+(\varepsilon \mathcal{E}(t), \tilde{\mathcal{E}}(t))_{\Omega}\right) \mathrm{d} t
$$

Lemma 4.7. The stability constants of Maxwell's equations can be defined as

$$
C_{L}=2 T \text {. }
$$

Proof. Due to the boundary conditions in $\mathcal{D}(L)$, we have $(\nabla \times \mathcal{E}(s), \mathcal{H}(s))_{\Omega}-(\nabla \times \mathcal{H}(s), \mathcal{E}(s))_{\Omega}=0$. Then, a straightforward use of the definition of the norm and of the initial condition shows for $(\mathcal{H}, \mathcal{E}) \in \mathcal{D}(L)$

$$
\begin{aligned}
\|(\mathcal{H}, \mathcal{E})\|_{H}^{2} & =\int_{0}^{T} \int_{0}^{t} \partial_{t}\left((\mu \mathcal{H}(s), \mathcal{H}(s))_{\Omega}+(\varepsilon \mathcal{E}(s), \mathcal{E}(s))_{\Omega}\right) \mathrm{d} s \mathrm{~d} t \\
& =2 \int_{0}^{T} \int_{0}^{t}\left(\left(\mu \partial_{t} \mathcal{H}(s), \mathcal{H}(s)\right)_{\Omega}+\left(\varepsilon \partial_{t} \mathcal{E}(s), \mathcal{E}(s)\right)_{\Omega}\right) \mathrm{d} s \mathrm{~d} t \\
& =2 \int_{0}^{T} \int_{0}^{t}\left((L(\mathcal{H}, \mathcal{E})(s),(\mu \mathcal{H}, \varepsilon \mathcal{E}))_{\Omega}-(\nabla \times \mathcal{E}(s), \mathcal{H}(s))_{\Omega}+(\nabla \times \mathcal{H}(s), \mathcal{E}(s))_{\Omega}\right) \mathrm{d} s \mathrm{~d} t \\
& =2 \int_{0}^{T} \int_{0}^{t}\left((L(\mathcal{H}, \mathcal{E})(s),(\mu \mathcal{H}, \varepsilon \mathcal{E}))_{\Omega} \mathrm{d} s \mathrm{~d} t \leq 2 T\|L(\mathcal{H}, \mathcal{E})\|_{H}\|(\mathcal{H}, \mathcal{E})\|_{H} .\right.
\end{aligned}
$$

The monochromatic Maxwell problem. Now we consider special monochromatic solutions of Maxwell's equation of the form $(\mathcal{H}, \mathcal{E})(x, t)=\exp (\mathrm{i} \omega t)(h(x), e(x))$ for given frequency $\omega$; this results in

$$
L(h, e)=\left(\mathrm{i} \omega h+\mu^{-1} \nabla \times e, \mathrm{i} \omega e-\varepsilon^{-1} \nabla \times h\right) .
$$


In analogy to the Helmholtz case, we show stability on

$$
\begin{aligned}
\mathcal{D}(L)=\left\{(h, e) \in \mathrm{H}(\operatorname{curl}, \Omega)^{2}:\right. & (\mu h, \nabla \psi)_{\Omega}=(\varepsilon e, \nabla \psi)_{\Omega}=0 \text { for all } \psi \in \mathrm{C}_{0}^{\infty}(\Omega), \\
& \left.\langle n \times h, \phi\rangle=\langle n \times e, n \times \phi\rangle \text { for all } \phi \in \mathrm{C}^{\infty}(\partial \Omega)^{3}\right\} .
\end{aligned}
$$

Smooth functions with $\nabla \cdot(\varepsilon e)=\nabla \cdot(\mu h)=0$ in $\Omega$ and Robin-type boundary condition $n \times h-(n \times e) \times n=0$ on $\partial \Omega$ are dense in $\mathcal{D}(L)$. We set $H$ as the closure of $\mathcal{D}(L)$ in $\mathrm{L}_{2}\left(\Omega, \mathbb{C}^{3} \times \mathbb{C}^{3}\right)$ with $\|(h, e)\|_{H}^{2}=(h, \mu h)_{\Omega}+(e, \varepsilon e)_{\Omega}$. Following the Helmholtz case, we consider domains with $x \cdot n(x) \geq \alpha>0$ for a.a. $x \in \partial \Omega$ and $|x| \leq R$ for $x \in \Omega$.

Lemma 4.8. The stability constants of the monochromatic Maxwell equation can be defined as

$$
C_{L}=2 \sqrt{\varepsilon \mu} R+\frac{(\mu+\varepsilon) R^{2}}{\alpha} .
$$

Proof. For the proof, we use the techniques in ([23] Thm. 3.3, [29] Thm. 5.4.5). We start with an upper bound for $\|n \times e\|_{\partial \Omega}$. For all smooth functions in $(h, e) \in \mathcal{D}(L)$, we find

$$
(L(h, e),(h, e))_{H}=(\mathrm{i} \omega \mu h+\nabla \times e, h)_{\Omega}+(\mathrm{i} \omega \varepsilon e-\nabla \times h, e)_{\Omega}=\mathrm{i} \omega\|(h, e)\|_{H}^{2}+(\nabla \times e, h)_{\Omega}-(\nabla \times h, e)_{\Omega} .
$$

Integration by parts then yields in terms of the boundary condition for the real part

$$
\operatorname{Re}(L(h, e),(h, e))_{H}=\operatorname{Re}\left((\nabla \times e, h)_{\Omega}-(\nabla \times h, e)_{\Omega}\right)=-\operatorname{Re}(n \times h, e)_{\partial \Omega}=-\|n \times e\|_{\partial \Omega}^{2} .
$$

This gives $\|n \times e\|_{\partial \Omega}^{2} \leq\|L(h, e)\|_{H}\|(h, e)\|_{H}$.

The rest of the proof is based on the two following identities which can be obtained by a straightforward computation

$$
\begin{gathered}
(x \times \bar{h}) \cdot(\nabla \times h)+(x \times h) \cdot(\nabla \times \bar{h})+h \cdot \bar{h}=\nabla \cdot((h \cdot \bar{h}) x)-\nabla \cdot((x \cdot h) \bar{h}) \\
+(\nabla \cdot \bar{h})(x \cdot h)-\nabla \cdot((x \cdot \bar{h}) h)+(\nabla \cdot h)(x \cdot h), \\
(x \times h) \cdot(n \times \bar{h})=x \cdot n|h|^{2}-(x \cdot h)(n \cdot \bar{h})
\end{gathered}
$$

see, e.g., Lemma 5.3.1 from [29], for (4.13b). Using $\nabla \cdot h=0$, we get in terms of the Gauss theorem

$$
2 \operatorname{Re}(\nabla \times h, x \times h)_{\Omega}+(h, h)_{\Omega}=\left(n \cdot x,|h|^{2}\right)_{\partial \Omega}-2 \operatorname{Re}(n \cdot h, x \cdot h)_{\partial \Omega}=2 \operatorname{Re}(n \times h, x \times h)_{\partial \Omega}-\left(n \cdot x,|h|^{2}\right)_{\partial \Omega} .
$$

Finally by means of $h \cdot(x \times \bar{e})=(h \times x) \cdot \bar{e}=-(x \times h) \cdot \bar{e}$, we can establish the following equality

$$
\begin{aligned}
& 2 \operatorname{Re}(L(h, e),(x \times \varepsilon e,-x \times \mu h))_{H} \\
& \quad=2 \operatorname{Re}\left((\mathrm{i} \omega \mu h, x \times \varepsilon e)_{\Omega}+(\nabla \times e, x \times \varepsilon e)_{\Omega}-(\mathrm{i} \omega \varepsilon e, x \times \mu h)_{\Omega}+(\nabla \times h, x \times \mu h)_{\Omega}\right) \\
& \quad=2 \mu \operatorname{Re}(\nabla \times h, x \times h)_{\Omega}+2 \varepsilon \operatorname{Re}(\nabla \times e, x \times e)_{\Omega} \\
& \quad=2 \mu \operatorname{Re}(n \times h, x \times h)_{\partial \Omega}-\mu\left(n \cdot x,|h|^{2}\right)_{\partial \Omega}-(h, \mu h)_{\Omega}+2 \varepsilon \operatorname{Re}(n \times e, x \times e)_{\partial \Omega}-\varepsilon\left(n \cdot x,|e|^{2}\right)_{\partial \Omega}-(e, \varepsilon e)_{\Omega} .
\end{aligned}
$$

Now we can use the definition of the boundary conditions and get

$$
\begin{aligned}
(h, \mu h)_{\Omega} & +(e, \varepsilon e)_{\Omega}+\mu\left(n \cdot x,|h|^{2}\right)_{\partial \Omega}+\varepsilon\left(n \cdot x,|e|^{2}\right)_{\partial \Omega} \\
& =-2 \operatorname{Re}(L(h, e),(x \times \varepsilon e,-x \times \mu h))_{H}+2 \mu \operatorname{Re}(n \times e, n \times(x \times h))_{\partial \Omega}+2 \varepsilon \operatorname{Re}(n \times e, x \times e)_{\partial \Omega} .
\end{aligned}
$$

Applying Young's inequality results in the estimate

$$
\begin{aligned}
\|(h, e)\|_{H}^{2}+\alpha & \mu h\left\|_{\partial \Omega}^{2}+\alpha \varepsilon\right\| e \|_{\partial \Omega}^{2} \\
& \leq 2\|L(h, e)\|_{H}\|(x \times \varepsilon e,-x \times \mu h)\|_{H}+2 \mu\|n \times e\|_{\partial \Omega}\|n \times(x \times h)\|_{\partial \Omega}+2 \varepsilon\|n \times e\|_{\partial \Omega}\|x \times e\|_{\partial \Omega} \\
& \leq 2 \sqrt{\varepsilon \mu} R\|L(h, e)\|_{H}\|(h, e)\|_{H}+2 \mu R\|n \times e\|_{\partial \Omega}\|h\|_{\partial \Omega}+2 \varepsilon R\|n \times e\|_{\partial \Omega}\|e\|_{\partial \Omega} \\
& \leq 2 \sqrt{\varepsilon \mu} R\|L(h, e)\|_{H}\|(h, e)\|_{H}+\frac{\mu R^{2}}{\alpha}\|n \times e\|_{\partial \Omega}^{2}+\alpha \mu\|h\|_{\partial \Omega}^{2}+\frac{\varepsilon R^{2}}{\alpha}\|n \times e\|_{\partial \Omega}^{2}+\alpha \varepsilon\|e\|_{\partial \Omega}^{2} .
\end{aligned}
$$


Observing that the $\mathrm{L}_{2}(\partial \Omega)$-terms cancel, we obtain by means of the upper bound for $\|n \times e\|_{\partial \Omega}^{2}$

$$
\|(h, e)\|_{H}^{2} \leq 2 \sqrt{\varepsilon \mu} R\|L(h, e)\|_{H}\|(h, e)\|_{H}+\frac{(\mu+\varepsilon) R^{2}}{\alpha}\|L(h, e)\|_{H}\|(h, e)\|_{H}
$$

This is shown for all sufficiently smooth functions satisfying (4.13) and tangential trace in $\mathrm{L}_{2}$; a density argument now yields the stability constant $C_{L}$ in $\mathcal{D}(L)$.

Remark 4.9. We note that $C_{L}$ does not depend on the wave number.

\section{The Continuous substruCturing Method}

In this section, we provide a Schur complement formulation of the problem. It gives rise to a symmetric positive definite formulation and is thus of special interest for efficient numerical solvers. To do so, we start with the introduction of a suitable partitioning and trace spaces, formulate the weak problem and apply a local static condensation.

We assume that we are in the abstract setting of Subsection 4.1. Then, for all $f \in H$ a unique solution $u^{\text {sol }} \in U$ of $L u^{\text {sol }}=f$ exists such that $\left\|u^{\text {sol }}\right\|_{U} \leq \sqrt{C_{L}^{2}+1}\|f\|_{H}$. We recall, that we use the graph norm $\|u\|_{U}^{2}=\|u\|_{H}^{2}+\|L u\|_{H}^{2}$ for $u \in U$. Moreover, let $U^{\mathrm{ad}}=\left\{v \in H: f \in H\right.$ exists such that $(v, L u)_{H}=(f, u)_{H}$ for all $u \in U\}$ be the adjoint space, and the Hilbert adjoint $L^{\text {ad }}$ is given by $(v, L u)_{H}=\left(L^{\text {ad }} v, u\right)_{H}$ for $v \in U^{\text {ad }}$.

Partitioning and product space. For a given disjoint partitioning $\Omega \backslash \Gamma_{\mathcal{T}}=\bigcup_{\tau \in \mathcal{T}} \tau$, we define the local spaces $H_{\tau}=\left\{\left.u\right|_{\tau}: u \in H\right\} \subset \mathrm{L}_{2}(\tau)^{M}$ for all $\tau \in \mathcal{T}$, and we assume that the restrictions $\left.L\right|_{\tau}$ and $\left.L^{\text {ad }}\right|_{\tau}$ of $L$ and its adjoint $L^{\text {ad }}$, respectively, are defined in the extended space $V_{\tau} \supset\left\{\left.u\right|_{\tau}: u \in U+U^{\text {ad }}\right\}$. We assume that $V_{\tau}$ is a Hilbert space with the graph norm $\|v\|_{V_{\tau}}^{2}=\|v\|_{H_{\tau}}^{2}+\left\|L^{\text {ad }} v\right\|_{H_{\tau}}^{2}$ of the adjoint operator. We will use $V_{\tau}$ as test space. In general, $V_{\tau}$ will not include boundary conditions. Associated with the local spaces is the product space $V=\prod_{\tau} V_{\tau}$ equipped with the norm $\|v\|_{V}^{2}=\sum_{\tau}\left\|v_{\tau}\right\|_{V_{\tau}}^{2}$.

Traces and trace norms. On $\tau$, we assume that trace spaces $\hat{V}_{\tau}$ and surjective trace mappings $\gamma_{\tau}: V_{\tau} \longrightarrow \hat{V}_{\tau}$ exist such that $\hat{V}_{\tau}$ is identified with the quotient spaces $V_{\tau} / \mathcal{N}\left(\gamma_{\tau}\right)$, and we use the corresponding trace norm

$$
\left\|\hat{v}_{\tau}\right\|_{\hat{V}_{\tau}}=\inf _{\gamma_{\tau} v_{\tau}=\hat{v}_{\tau}}\left\|v_{\tau}\right\|_{V_{\tau}}
$$

Associated with the trace mapping $\gamma_{\tau}$ is the adjoint trace $\gamma_{\tau}^{\text {ad }}: V_{\tau} \longrightarrow \hat{V}_{\tau}^{\prime}$ defined by

$$
\left(L v_{\tau}, \tilde{v}_{\tau}\right)_{H_{\tau}}=\left(v_{\tau}, L^{\mathrm{ad}} \tilde{v}_{\tau}\right)_{H_{\tau}}+\left\langle\gamma_{\tau} v_{\tau}, \gamma_{\tau}^{\mathrm{ad}} \tilde{v}_{\tau}\right\rangle, \quad v_{\tau}, \tilde{v}_{\tau} \in V_{\tau} .
$$

The embedding $U \subset V$ allows to define $\hat{U}=\gamma_{\Gamma}(U)$, where $\gamma_{\Gamma}: V \longrightarrow \prod_{\tau} \hat{V}_{\tau}$ is the product trace mapping. We observe for the kernel of $\gamma_{\Gamma}$ that $\mathcal{N}\left(\gamma_{\Gamma}\right) \subset U$. The trace space $\hat{U}$ will be identified with the quotient space $\hat{U}=U / \mathcal{N}\left(\gamma_{\Gamma}\right)$ associated with the norm

$$
\|\hat{u}\|_{\hat{U}}=\inf _{\gamma_{\Gamma} u=\hat{u}}\|u\|_{U}
$$

For $u \in U$ and $\hat{u}=\gamma_{\Gamma} u$ we set $\hat{u}_{\tau}=\gamma_{\tau} u$. Then, we have for $\hat{v}_{\tau} \in V_{\tau}$ and $\hat{u}=\left(\hat{u}_{\tau}\right) \in \hat{U}$ the estimates $\left\|\gamma_{\tau} v_{\tau}\right\|_{\hat{V}_{\tau}} \leq\left\|v_{\tau}\right\|_{V_{\tau}}$ and

$$
\begin{aligned}
\left|\sum_{\tau}\left\langle\hat{u}_{\tau}, \gamma_{\tau}^{\mathrm{ad}} v_{\tau}\right\rangle\right| & =\inf _{\gamma_{\Gamma} u=\hat{u}}\left|\sum_{\tau}\left\langle\gamma_{\tau} u, \gamma_{\tau}^{\mathrm{ad}} v_{\tau}\right\rangle\right|=\inf _{\gamma_{\Gamma} u=\hat{u}}\left|\sum_{\tau}\left(L u, v_{\tau}\right)_{H_{\tau}}-\left(u, L^{\mathrm{ad}} v_{\tau}\right)_{H_{\tau}}\right| \\
& \leq \inf _{\gamma_{\Gamma} u=\hat{u}} \sum_{\tau}\left(\|L u\|_{H_{\tau}}\left\|v_{\tau}\right\|_{H_{\tau}}+\|u\|_{H_{\tau}}\left\|L^{\mathrm{ad}} v_{\tau}\right\|_{H_{\tau}}\right) \\
& \leq \inf _{\gamma_{\Gamma} u=\hat{u}}\left(\sum_{\tau}\|L u\|_{H_{\tau}}^{2}+\|u\|_{H_{\tau}}^{2}\right)^{\frac{1}{2}}\left(\sum_{\tau}\left\|v_{\tau}\right\|_{H_{\tau}}^{2}+\left\|L^{\mathrm{ad}} v_{\tau}\right\|_{H_{\tau}}^{2}\right)^{\frac{1}{2}} \\
& \leq \inf _{\gamma_{\Gamma} u=\hat{u}}\|u\|_{U}\|v\|_{V}=\|\hat{u}\|_{\hat{U}}\|v\|_{V} .
\end{aligned}
$$


A weak formulation. For given $f \in H$, we set $F_{\tau} \in V_{\tau}^{\prime}$ and $F \in V^{\prime}$ by $\left\langle F_{\tau}, v\right\rangle=(f, v)_{H_{\tau}}$ and $\langle F, v\rangle=(f, v)_{H}$, respectively. In a next step, we define the local bilinear forms $B_{\tau} \in \mathcal{L}\left(H_{\tau}, V_{\tau}^{\prime}\right)$ and $\hat{B}_{\tau} \in \mathcal{L}\left(\hat{U}, V_{\tau}^{\prime}\right)$ by

$$
\left\langle B_{\tau} u, v\right\rangle=\left(u, L^{\mathrm{ad}} v\right)_{H_{\tau}}, \quad\left\langle\hat{B}_{\tau} \hat{u}, v\right\rangle=\left\langle\hat{u}_{\tau}, \gamma_{\tau}^{\mathrm{ad}} v\right\rangle,
$$

and observe that $(L u, v)_{H_{\tau}}=\left\langle B_{\tau} u, v\right\rangle+\left\langle\hat{B}_{\tau} \gamma_{\tau} u, v\right\rangle$. For the global operator $B \in \mathcal{L}\left(H \times \hat{U}, V^{\prime}\right)$ given by $\langle B(u, \hat{u}), v\rangle=\sum_{\tau}\left\langle B_{\tau} u_{\tau}+\hat{B}_{\tau} \hat{u}, v_{\tau}\right\rangle$, we find that the solution $u^{\text {sol }} \in U$ of $L u^{\text {sol }}=f$ and its trace $\hat{u}^{\text {sol }}=\gamma_{\Gamma} u^{\text {sol }}$ satisfy

$$
B\left(u^{\mathrm{sol}}, \hat{u}^{\mathrm{sol}}\right)=F .
$$

This observation motivates our interest to focus on the properties of $B(\cdot, \cdot)$. The following lemma shows that (5.2) has a unique solution and thus is equivalent to our original problem.

Lemma 5.1. The operator $B \in \mathcal{L}\left(H \times \hat{U}, V^{\prime}\right)$ is bounded by

$$
|\langle B(u, \hat{u}), v\rangle| \leq C_{B}\|(u, \hat{u})\|_{H \times \hat{U}}\|v\|_{V}, \quad(u, \hat{u}) \in H \times \hat{U}
$$

with $C_{B}=\sqrt{2}$, and for given $f \in H$ a unique solution of (5.2) exists.

Proof. Using (5.1) we obtain for $(u, \hat{u}) \in H \times \hat{U}$

$$
\begin{aligned}
\left|\sum_{\tau}\left\langle B_{\tau} u_{\tau}, v_{\tau}\right\rangle\right| & \leq \sum_{\tau}\left\|u_{\tau}\right\|_{H_{\tau}}\left\|L^{\mathrm{ad}} v_{\tau}\right\|_{H_{\tau}} \leq\|u\|_{H}\|v\|_{V}, \\
\left|\sum_{\tau}\left\langle\hat{B}_{\tau} \hat{u}, v_{\tau}\right\rangle\right| & =\left|\sum_{\tau}\left\langle\hat{u}, \gamma_{\tau}^{\mathrm{ad}} v_{\tau}\right\rangle\right| \leq\|\hat{u}\|_{\hat{U}}\|v\|_{V},
\end{aligned}
$$

and thus $|\langle B(u, \hat{u}), v\rangle| \leq\left(\|u\|_{H}+\|\hat{u}\|_{\hat{U}}\right)\|v\|_{V} \leq \sqrt{2}\|(u, \hat{u})\|_{H \times \hat{U}}\|v\|_{V}$.

The solution $u^{\text {sol }} \in U$ of $L u^{\text {sol }}=f$ is unique, and we have $B\left(u^{\text {sol }}, \gamma_{\Gamma} u^{\text {sol }}\right)=F$ by construction. Thus, it is sufficient to show that $B$ is injective. Let $(u, \hat{u}) \in U \times \hat{U}$ be a solution of the homogeneous problem $B(u, \hat{u})=0$. In the first step, we test with $v \in \mathcal{N}\left(\gamma_{\Gamma}\right) \subset U$ which yields

$$
0=\langle B(u, \hat{u}), v\rangle=\left(u, L^{\mathrm{ad}} v\right)_{H}=(L u, v)_{H} .
$$

Since $\mathcal{N}\left(\gamma_{\Gamma}\right)$ is dense in $H$, this implies $L u=0$ and since $L$ is injective, we obtain $u=0$. Then, we have $0=\langle B(0, \hat{u}), v\rangle=\sum_{\tau}\left\langle\hat{B}_{\tau} \hat{u}, v_{\tau}\right\rangle$ for all $v \in V$, with implies $\hat{u}=0$, since $\hat{B}_{\tau}$ is injective. This shows that $B$ is injective on $U \times \hat{U}$, and since $U$ is dense in $H$ and $B$ is continuous, $B$ is also injective on $H \times \hat{U}$.

The Schur complement. For the solution of the equation $B\left(u^{\mathrm{sol}}, \hat{u}^{\mathrm{sol}}\right)=F$ we now construct a Schur complement problem. To do so, we introduce self-adjoint and positive operators $A_{\tau} \in \mathcal{L}\left(V_{\tau}, V_{\tau}^{\prime}\right)$ and $A \in \mathcal{L}\left(V, V^{\prime}\right)$ by

$$
\left\langle A_{\tau} v_{\tau}, \tilde{v}_{\tau}\right\rangle=\left(v_{\tau}, \tilde{v}_{\tau}\right)_{H_{\tau}}+\left(L^{\mathrm{ad}} v_{\tau}, L^{\mathrm{ad}} \tilde{v}_{\tau}\right)_{H_{\tau}}, \quad\langle A v, \tilde{v}\rangle=\sum_{\tau}\left\langle A_{\tau} v_{\tau}, \tilde{v}_{\tau}\right\rangle,
$$

and the Schur complement $S=B^{\prime} A^{-1} B \in \mathcal{L}\left(H \times \hat{U}, H^{\prime} \times \hat{U}^{\prime}\right)$. This gives

$$
S\left(u^{\mathrm{sol}}, \hat{u}^{\mathrm{sol}}\right)=B^{\prime} A^{-1} F \text {. }
$$

We now show that this problem is well-posed using the abstract theory of Section 3 with $Y=H \times \hat{U}$.

Lemma 5.2. We have for all $(u, \hat{u}) \in H \times \hat{U}$

$$
c_{S}\|(u, \hat{u})\|_{H \times \hat{U}}^{2} \leq\langle S(u, \hat{u}),(u, \hat{u})\rangle \leq C_{S}\|(u, \hat{u})\|_{H \times \hat{U}}^{2}
$$

with $c_{S}=\left(4 C_{L}^{2}+2\right)^{-1}$ and $C_{S}=2$. 
Proof. We start with the proof of the upper bound. We have $\langle A v, v\rangle=\|v\|_{V}^{2},\left\langle F, A^{-1} F\right\rangle=\|F\|_{V^{\prime}}^{2}$, and

$$
\langle S(u, \hat{u}),(u, \hat{u})\rangle=\left\langle B(u, \hat{u}), A^{-1} B(u, \hat{u})\right\rangle=\|B(u, \hat{u})\|_{V^{\prime}}^{2}=\sup _{v \in V} \frac{|\langle B(u, \hat{u}), v\rangle|^{2}}{\|v\|_{V}^{2}} .
$$

Thus, Lemma 5.1 yields the upper bound $C_{S}=C_{B}^{2}=2$.

To show the lower bound, we use that for all $v \in V \subset H$ a function $u_{v} \in U$ with $L u_{v}=v$ exists. Using the definition of the norm in $U$ and $\hat{U}$, we get

$$
\begin{aligned}
\left\|\left(u_{v}, \gamma_{\Gamma} u_{v}\right)\right\|_{H \times \hat{U}}^{2}=\left\|u_{v}\right\|_{H}^{2}+\left\|\gamma_{\Gamma} u_{v}\right\|_{\hat{U}}^{2} & \leq\left\|u_{v}\right\|_{H}^{2}+\left\|u_{v}\right\|_{U}^{2}=2\left\|u_{v}\right\|_{H}^{2}+\left\|L u_{v}\right\|_{H}^{2} \\
& \leq\left(2 C_{L}^{2}+1\right)\left\|L u_{v}\right\|_{H}^{2}=\left(2 C_{L}^{2}+1\right)\|v\|_{H}^{2} .
\end{aligned}
$$

This observation and $\left\langle B\left(u_{v}, \gamma_{\Gamma} u_{v}\right), v\right\rangle=\left(L u_{v}, v\right)_{H}=\|v\|_{H}^{2}$ yield

$$
\sup _{(u, \hat{u}) \in H \times \hat{U}} \frac{|\langle B(u, \hat{u}), v\rangle|}{\|(u, \hat{u})\|_{H \times \hat{U}}} \geq \frac{\left\langle B\left(u_{v}, \gamma_{\Gamma} u_{v}\right), v\right\rangle}{\left\|\left(u_{v}, \gamma_{\Gamma} u_{v}\right)\right\|_{H \times \hat{U}}} \geq \frac{1}{\sqrt{2 C_{L}^{2}+1}}\|v\|_{H} .
$$

Moreover testing with $(u, \hat{u})=\left(L^{\text {ad }} v, 0\right)$, a second lower bound is obtained

$$
\sup _{(u, \hat{u}) \in H \times \hat{U}} \frac{|\langle B(u, \hat{u}), v\rangle|}{\|(u, \hat{u})\|_{H \times \hat{U}}} \geq \frac{\left\langle B\left(L^{\mathrm{ad}} v, 0\right), v\right\rangle}{\left\|L^{\mathrm{ad}} v\right\|_{H}}=\left\|L^{\mathrm{ad}} v\right\|_{H} .
$$

Combining these two estimates gives

$$
\sup _{(u, \hat{u}) \in H \times \hat{U}} \frac{|\langle B(u, \hat{u}), v\rangle|}{\|(u, \hat{u})\|_{H \times \hat{U}}} \geq \max \left\{\frac{\|v\|_{H}}{\sqrt{2 C_{L}^{2}+1}},\left\|L^{\mathrm{ad}} v\right\|_{H}\right\} \geq \frac{\sqrt{\|v\|_{H}^{2}+\left\|L^{\mathrm{ad}} v\right\|_{H}^{2}}}{\sqrt{2} \sqrt{2 C_{L}^{2}+1}}=\frac{1}{\sqrt{4 C_{L}^{2}+2}}\|v\|_{V} .
$$

Recalling that $B$ is injective, the lower bound is now obtained by duality, see, e.g., [6], Lemma 4.4.2.

The skeleton reduction. Finally we eliminate $u^{\text {sol }}$ and obtain a positive definite system for the trace $\hat{u}^{\text {sol }}$. We point out that $u^{\text {sol }}$ can be recovered locally from $\hat{u}^{\text {sol }}$. This is an attractive feature for the discrete setting.

Inserting $\hat{u}=0$ gives for the local Schur complement $S_{\tau}=B_{\tau}^{\prime} A_{\tau}^{-1} B_{\tau}$ the estimate $c_{S}\left\|u_{\tau}\right\|_{H_{\tau}}^{2} \leq\left\langle S_{\tau} u_{\tau}, u_{\tau}\right\rangle$. Thus, we can construct the local projection $P_{\tau}=\mathrm{id}_{V_{\tau}}-A_{\tau}^{-1} B_{\tau} S_{\tau}^{-1} B_{\tau}^{\prime}$ on $\mathcal{N}\left(B_{\tau}^{\prime}\right)$ with $P_{\tau} A_{\tau}^{-1} B_{\tau}=0$. Moreover, we have $A_{\tau} P_{\tau}=P_{\tau}^{\prime} A_{\tau}$, i.e., $P_{\tau}$ is an orthogonal projection in $V_{\tau}$.

We use the projection to eliminate the local solutions $u_{\tau}^{\text {sol }} \in H_{\tau}$ and thus for the reduction to the skeleton values $\hat{U}$. We obtain from $B_{\tau} u^{\mathrm{sol}}+\hat{B}_{\tau} \hat{u}^{\mathrm{sol}}=F_{\tau}$ the local equation $P_{\tau} A_{\tau}^{-1} \hat{B}_{\tau} \hat{u}^{\mathrm{sol}}=P_{\tau} A_{\tau}^{-1} F_{\tau}$. Setting on the skeleton $\hat{S}=\sum_{\tau} \hat{B}_{\tau}^{\prime} P_{\tau} A_{\tau}^{-1} \hat{B}_{\tau}$ and $\hat{F}=\sum_{\tau} \hat{B}_{\tau}^{\prime} P_{\tau} A_{\tau}^{-1} F_{\tau}$, the global equation for $u^{\text {sol }}$ can be rewritten as

$$
\hat{S} \hat{u}^{\mathrm{sol}}=\hat{F} .
$$

Lemma 5.3. We have $c_{S}\|\hat{u}\|_{\hat{U}}^{2} \leq\langle\hat{S} \hat{u}, \hat{u}\rangle \leq\|\hat{u}\|_{\hat{U}}^{2}$ for $\hat{u} \in \hat{U}$.

Proof. For given $\hat{u} \in \hat{U}$ and the special choice $u_{\tau}=-S_{\tau}^{-1} B_{\tau}^{\prime} A_{\tau}^{-1} \hat{B}_{\tau} \hat{u}$, we have

$$
B_{\tau} u_{\tau}+\hat{B}_{\tau} \hat{u}=\left(-B_{\tau} S_{\tau}^{-1} B_{\tau}^{\prime} A_{\tau}^{-1}+\operatorname{id}_{V_{\tau}^{\prime}}\right) \hat{B}_{\tau} \hat{u}=P_{\tau}^{\prime} \hat{B}_{\tau} \hat{u} .
$$

Using $P_{\tau} A_{\tau}^{-1} P_{\tau}^{\prime}=P_{\tau} A_{\tau}^{-1}$, we obtain for $u=\left(u_{\tau}\right)$

$$
\langle S(u, \hat{u}),(u, \hat{u})\rangle=\sum_{\tau}\left\langle B_{\tau} u_{\tau}+\hat{B}_{\tau} \hat{u}, A_{\tau}^{-1}\left(B_{\tau} u_{\tau}+\hat{B}_{\tau} \hat{u}\right)\right\rangle=\sum_{\tau}\left\langle P_{\tau}^{\prime} \hat{B}_{\tau} \hat{u}, A_{\tau}^{-1} P_{\tau}^{\prime} \hat{B}_{\tau} \hat{u}\right\rangle=\langle\hat{S} \hat{u}, \hat{u}\rangle .
$$


Then, the lower bound $c_{S}\|\hat{u}\|_{\hat{U}}^{2} \leq c_{S}\|(u, \hat{u})\|_{H \times \hat{U}}^{2} \leq\langle\hat{S} \hat{u}, \hat{u}\rangle$ follows from Lemma 5.2, and the upper bound follows from (5.1) by

$$
\begin{aligned}
\langle\hat{S} \hat{u}, \hat{u}\rangle & =\sum_{\tau}\left\langle\hat{B}_{\tau} \hat{u}, P_{\tau} A_{\tau}^{-1} \hat{B}_{\tau} \hat{u}\right\rangle \leq \sum_{\tau}\left\|\hat{B}_{\tau} \hat{u}\right\|_{V_{\tau}^{\prime}}\left\|P_{\tau} A_{\tau}^{-1} \hat{B}_{\tau} \hat{u}\right\|_{V_{\tau}} \\
& \leq \sum_{\tau}\left\|\hat{B}_{\tau} \hat{u}\right\|_{V_{\tau}^{\prime}}^{2} \leq \sum_{\tau\left\|v_{\tau}\right\|_{V_{\tau}}=1}\left\langle\hat{u}_{\tau}, \gamma_{\tau}^{\text {ad }} v_{\tau}\right\rangle^{2} \leq\|\hat{u}\|_{\hat{U}}^{2} .
\end{aligned}
$$

This shows that $\hat{S}$ is positive and the equation (5.4) has a unique solution $\hat{u}^{\text {sol }}$. Then, the local solutions $u_{\tau}^{\text {sol }}=S_{\tau}^{-1} B_{\tau}^{\prime} A_{\tau}^{-1}\left(F_{\tau}-\hat{B}_{\tau} \hat{u}^{\text {sol }}\right)$ are determined by the skeleton solution and the right-hand side.

\section{The Discrete SUbstruCtURING Method}

In this section, we firstly proceed exactly as in the continuous setting and then comment on inf-sup stability and preconditioning. Since the Schur complement equation for the continuous problem corresponds to a saddle point problem, we need a uniform inf-sup stable discretization. Let $H_{\tau, h} \subset H_{\tau}$ be local finite element spaces, and let $\hat{U}_{h} \subset \hat{U}$ be the trace space of a conforming finite element space. Then, we assume that discrete spaces $V_{\tau, h} \subset V_{\tau}$ and $\beta_{0} \in(0,1)$ exists satisfying for all $\left(u_{\tau, h}, \hat{u}_{h}\right) \in H_{\tau, h} \times \hat{U}_{h}$ and $\tau \in \mathcal{T}$

$$
\sup _{v_{\tau, h} \in V_{\tau, h}} \frac{\left|\left\langle B_{\tau} u_{\tau, h}+\hat{B}_{\tau} \hat{u}_{h}, v_{\tau, h}\right\rangle\right|}{\left\|v_{\tau, h}\right\|_{V_{\tau}}} \geq \beta_{0} \sup _{v_{\tau} \in V_{\tau}} \frac{\left|\left\langle B_{\tau} u_{\tau, h}+\hat{B}_{\tau} \hat{u}_{h}, v_{\tau}\right\rangle\right|}{\left\|v_{\tau}\right\|_{V_{\tau}}} .
$$

Let $B_{\tau, h}, \hat{B}_{\tau, h}, A_{\tau, h}$, and $F_{\tau, h}$ be the Galerkin approximations of $B_{\tau}, \hat{B}_{\tau}, A_{\tau}$, and $F_{\tau}$, i.e., for $u_{\tau, h} \in H_{\tau, h}$, $\hat{u}_{\tau, h} \in \hat{U}_{h}$, and $v_{\tau, h}, \tilde{v}_{\tau, h} \in V_{\tau, h}$ we have

$$
\begin{aligned}
\left\langle B_{\tau, h} u_{\tau, h}, v_{\tau, h}\right\rangle & =\left\langle B_{\tau} u_{\tau, h}, v_{\tau, h}\right\rangle, & & \left\langle\hat{B}_{\tau, h} \hat{u}_{\tau, h}, v_{\tau, h}\right\rangle=\left\langle\hat{B}_{\tau} \hat{u}_{\tau, h}, v_{\tau, h}\right\rangle, \\
\left\langle A_{\tau, h} v_{\tau, h}, \tilde{v}_{\tau, h}\right\rangle & =\left\langle A_{\tau} v_{\tau, h}, \tilde{v}_{\tau, h}\right\rangle, & & \left\langle F_{\tau, h}, v_{\tau, h}\right\rangle=\left\langle F_{\tau}, v_{\tau, h}\right\rangle .
\end{aligned}
$$

As in the continuous setting, we define the spaces $H_{h}=\prod_{\tau} H_{\tau, h}$ and $V_{h}=\prod_{\tau} V_{\tau, h}$. The Galerkin approximations of $B, A$, and $F$ are denoted by $B_{h} \in \mathcal{L}\left(H_{h} \times \hat{U}_{h}, V_{h}\right), A_{h} \in \mathcal{L}\left(V_{h}, V_{h}^{\prime}\right)$, and $F_{h} \in V_{h}^{\prime}$. Since $A_{h}$ is symmetric and positive, the discrete approximation $S_{h}=B_{h}^{\prime} A_{h}^{-1} B_{h} \in \mathcal{L}\left(H_{h} \times \hat{U}_{h}, H_{h}^{\prime} \times \hat{U}_{h}^{\prime}\right)$ of $S$ is well-defined.

A direct approximation of the equation $B\left(u^{\mathrm{sol}}, \hat{u}^{\mathrm{sol}}\right)=F$ requires that the discrete solution space $H_{h} \times \hat{U}_{h}$ and the test space have the same dimension. This can be achieved by selecting the test space $A_{h}^{-1} B_{h}\left(H_{h} \times \hat{U}_{h}\right) \subset V_{h}$, or equivalently, by the solution of the discrete Schur complement problem given by $S_{h}\left(u_{h}^{\text {sol }}, \hat{u}_{h}^{\text {sol }}\right)=B_{h}^{\prime} A_{h}^{-1} F_{h}$.

We now show that the local inf-sup stability guarantees that $S_{h}$ is uniformly positive in $Y_{h}=H_{h} \times \hat{U}_{h}$, so that a unique solution of the Schur complement problem exists.

Lemma 6.1. We have for all $\left(u_{h}, \hat{u}_{h}\right) \in H_{h} \times \hat{U}_{h}$

$$
c_{S} \beta_{0}^{2}\left\|\left(u_{h}, \hat{u}_{h}\right)\right\|_{H \times \hat{U}}^{2} \leq\left\langle S_{h}\left(u_{h}, \hat{u}_{h}\right),\left(u_{h}, \hat{u}_{h}\right)\right\rangle \leq C_{S}\left\|\left(u_{h}, \hat{u}_{h}\right)\right\|_{H \times \hat{U}}^{2} .
$$

Proof. The upper bound follows from the continuity of the operator $B$. More precisely, we get

$$
\sup _{v_{h} \in V_{h}} \frac{\left|\left\langle B_{h}\left(u_{h}, \hat{u}_{h}\right), v_{h}\right\rangle\right|}{\left\|v_{h}\right\|_{V}} \leq \sup _{v \in V} \frac{\left|\left\langle B\left(u_{h}, \hat{u}_{h}\right), v\right\rangle\right|}{\|v\|_{V}} \leq C_{B}\|(u, \hat{u})\|_{H \times \hat{U}} .
$$

By construction of $V_{\tau, h}$, we have for all $\left(u_{h}, \hat{u}_{h}\right) \in H_{h} \times \hat{U}_{h}$

$$
\begin{aligned}
\left\langle S_{h}\left(u_{h}, \hat{u}_{h}\right),\left(u_{h}, \hat{u}_{h}\right)\right\rangle & =\sup _{v_{h} \in V_{h}} \frac{\left|\left\langle B_{h}\left(u_{h}, \hat{u}_{h}\right), v_{h}\right\rangle\right|^{2}}{\left\|v_{h}\right\|_{V}^{2}}=\sum_{\tau} \sup _{v_{\tau, h} \in V_{\tau, h}} \frac{\left|\left\langle B_{\tau, h} u_{\tau, h}+\hat{B}_{\tau, h} \hat{u}_{h}, v_{\tau, h}\right\rangle\right|^{2}}{\left\|v_{\tau, h}\right\|_{V_{\tau}}^{2}} \\
& \geq \beta_{0}^{2} \sum_{\tau} \sup _{v_{\tau} \in V_{\tau}} \frac{\left|\left\langle B_{\tau} u_{\tau, h}+B_{\tau} \hat{u}_{h}, v_{\tau}\right\rangle\right|^{2}}{\left\|v_{\tau}\right\|_{V_{\tau}}^{2}}=\beta_{0}^{2}\left\langle S\left(u_{h}, \hat{u}_{h}\right),\left(u_{h}, \hat{u}_{h}\right)\right\rangle,
\end{aligned}
$$

so that the lower bound is obtained from Lemma 5.2. 
Lemma 6.1 allows us to formulate an a priori bound for $\left(u^{\mathrm{sol}}, \hat{u}^{\mathrm{sol}}\right)-\left(u_{h}^{\mathrm{sol}}, \hat{u}_{h}^{\mathrm{sol}}\right)$.

Theorem 6.2. We have the a priori bound

$$
\left\|\left(u^{\mathrm{sol}}, \hat{u}^{\mathrm{sol}}\right)-\left(u_{h}^{\mathrm{sol}}, \hat{u}_{h}^{\mathrm{sol}}\right)\right\|_{H \times \hat{U}} \leq \frac{\sqrt{C_{S}}}{\beta_{0} \sqrt{C_{S}}} \inf _{\left(u_{h}, \hat{u}_{h}\right) \in H_{h} \times \hat{U}_{h}}\left\|\left(u^{\mathrm{sol}}, \hat{u}^{\mathrm{sol}}\right)-\left(u_{h}, \hat{u}_{h}\right)\right\|_{H \times \hat{U}}
$$

Proof. Combining (3.3) with Lemmas 5.2 and 6.1, see also Theorem 2.1 from [20], gives the a priori bound.

Remark 6.3. We note that Theorem 6.2 can be regarded as an extension of Céa's lemma to the more general setting of first order systems. More precisely, the stability and the inf-sup constants of first order systems play the same role as the continuity and coercivity constants for Céa's lemma, respectively. For parameter dependent problems, the ratio $\sqrt{C_{S}} /\left(\sqrt{c_{S}} \beta_{0}\right)=2 \sqrt{C_{L}^{2}+1} / \beta_{0}$ has to be uniformly bounded to obtain robust estimates with respect to the parameter space.

The skeleton reduction. For the elimination of the inner degrees of freedom, we proceed as in the continuous case. From Lemma 6.1 we obtain for the discrete local Schur complement $S_{\tau, h}=B_{\tau, h}^{\prime} A_{\tau, h}^{-1} B_{\tau, h}$ the estimate $c_{S} \beta_{0}^{2}\left\|u_{\tau, h}\right\|_{H_{\tau}}^{2} \leq\left\langle S_{\tau, h} u_{\tau, h}, u_{\tau, h}\right\rangle$. Thus, the local operators $S_{\tau, h}$ can be inverted, and we can define the local projections $P_{\tau, h}=\mathrm{id}_{V_{\tau, h}}-A_{\tau, h}^{-1} B_{\tau, h} S_{\tau, h}^{-1} B_{\tau, h}^{\prime}$ on $\mathcal{N}\left(B_{\tau, h}^{\prime}\right)$ and

$$
\hat{S}_{h}=\sum_{\tau} \hat{B}_{\tau, h}^{\prime} P_{\tau, h} A_{\tau, h}^{-1} \hat{B}_{\tau, h}, \quad \hat{F}_{h}=\sum_{\tau} \hat{B}_{\tau, h}^{\prime} P_{\tau, h} A_{\tau, h}^{-1} F_{\tau, h} .
$$

Analogously to Lemma 5.3, we obtain spectral bounds

$$
c_{S} \beta_{0}^{2}\left\|\hat{u}_{h}\right\|_{\hat{U}}^{2} \leq\left\langle\hat{S}_{h} \hat{u}_{h}, \hat{u}_{h}\right\rangle \leq\left\|\hat{u}_{h}\right\|_{\hat{U}}^{2}, \quad \hat{u}_{h} \in \hat{U}_{h} .
$$

This shows that $\hat{S}_{h}$ is positive, and $\hat{u}_{h}^{\text {sol }} \in \hat{U}_{h}$ is the unique solution of

$$
\hat{S}_{h} \hat{u}_{h}^{\text {sol }}=\hat{F}_{h} .
$$

Then, local computations give $u_{\tau, h}^{\text {sol }}=S_{\tau, h}^{-1} B_{\tau, h}^{\prime} A_{\tau, h}^{-1}\left(F_{\tau, h}-\hat{B}_{\tau, h} \hat{u}_{h}^{\text {sol }}\right)$ for all $\tau$.

Inf-sup stability. So far we have been assuming the existence of a uniform discrete inf-sup constant. Here, we derive a simple criterion and show that inf-sup stability can be achieved if $V_{h}$ is large enough.

We start with the explicit evaluation of the supremum. For given $\left(u_{\tau, h}, \hat{u}_{h}\right) \in H_{\tau, h} \times \hat{U}_{h}$, we define locally $F_{\tau}=B_{\tau} u_{\tau, h}+\hat{B}_{\tau} \hat{u}_{h} \in V_{\tau}^{\prime}$ and $F_{\tau, h}=B_{\tau, h} u_{\tau, h}+\hat{B}_{\tau, h} \hat{u}_{h} \in V_{\tau, h}^{\prime}$, and we observe

$$
\begin{aligned}
\left\|A_{\tau, h}^{-1} F_{\tau, h}\right\|_{V_{\tau}} & =\sqrt{\left\langle A_{\tau, h} A_{\tau, h}^{-1} F_{\tau, h}, A_{\tau, h}^{-1} F_{\tau, h}\right\rangle}=\sqrt{\left\langle F_{\tau, h}, A_{\tau, h}^{-1} F_{\tau, h}\right\rangle} \\
& =\sup _{v_{\tau, h} \in V_{\tau, h}} \frac{\left|\left\langle B_{\tau, h} u_{\tau, h}+\hat{B}_{\tau, h} \hat{u}_{h}, v_{\tau, h}\right\rangle\right|}{\left\|v_{\tau, h}\right\|_{V_{\tau}}}=\sup _{v_{\tau, h} \in V_{\tau, h}} \frac{\left|\left\langle B_{\tau} u_{\tau, h}+\hat{B}_{\tau} \hat{u}_{h}, v_{\tau, h}\right\rangle\right|}{\left\|v_{\tau, h}\right\|_{V_{\tau}}} \\
& \leq \sup _{v_{\tau} \in V_{\tau}} \frac{\left|\left\langle B_{\tau} u_{\tau, h}+B_{\tau} \hat{u}_{h}, v_{\tau}\right\rangle\right|}{\left\|v_{\tau}\right\|_{V_{\tau}}}=\sqrt{\left\langle F_{\tau}, A_{\tau}^{-1} F_{\tau}\right\rangle}=\left\|A_{\tau}^{-1} F_{\tau}\right\|_{V_{\tau}} .
\end{aligned}
$$

Thus, uniform inf-sup stability is obtained if we can show $\left\|A_{\tau, h}^{-1} F_{\tau, h}\right\|_{V_{\tau}} \geq \beta_{0}\left\|A_{\tau}^{-1} F_{\tau}\right\|_{V_{\tau}}$ for some $\beta_{0} \in(0,1)$ independent of the mesh size. For a more detailed discussion, we define the spaces $W_{\tau}=A_{\tau}^{-1}\left(B_{\tau} H_{\tau, h}+\hat{B}_{\tau} \hat{U}_{h}\right)$ and $W_{\tau, h}=A_{\tau, h}^{-1}\left(B_{\tau, h} H_{\tau, h}+\hat{B}_{\tau, h} \hat{U}_{h}\right)$. Note that $\operatorname{dim}\left(W_{\tau}\right) \leq \operatorname{dim}\left(H_{\tau, h}\right)+\operatorname{dim}\left(\hat{U}_{h}\right)$ in our applications. An obvious requirement for inf-sup stability is $\operatorname{dim}\left(W_{\tau, h}\right)=\operatorname{dim}\left(W_{\tau}\right)$. Since we have $\operatorname{dim}\left(W_{\tau, h}\right) \leq \operatorname{dim}\left(V_{\tau, h}\right)$, this implies that $V_{\tau, h}$ has to be large enough.

Now we discuss two cases. In the first case, we assume that $W_{\tau}$ can be determined explicitly. Then, we can compute $\beta_{0, \tau}>0$ for every $\tau$ by a simple eigenvalue analysis in $\mathbb{R}^{\operatorname{dim}\left(W_{\tau}\right)}$. For an affine family of subdomains $\tau$ 
this reduces to an eigenvalue analysis on a reference element. In this case, a suitable Fortin mapping can explicitly be constructed, see [20] for the Poisson problem and for linear elasticity.

For the more general case, we assume that $V_{\tau, h, p} \subset V_{\tau}, p \in \mathcal{P}$, is a dense family of discrete subspaces, so that the orthogonal projections $P_{\tau, h, p} \in \mathcal{L}\left(W_{\tau}, V_{\tau, h, p}\right)$ satisfy $\lim _{p \in \mathcal{P}}\left\|P_{\tau, h, p}-\operatorname{id}_{W_{\tau}}\right\|_{\mathcal{L}\left(W_{\tau}, V_{\tau}\right)}=0$. Recalling that $W_{\tau}$ is finite dimensional, for each $\beta_{0} \in(0,1)$ there exists a $p \in \mathcal{P}$ such that

$$
\inf _{v_{\tau, h, p} \in V_{\tau, h, p}}\left\|v_{\tau, h, p}-w_{\tau}\right\|_{V_{\tau}} \leq\left(1-\beta_{0}\right)\left\|w_{\tau}\right\|_{V_{\tau}}, \quad w_{\tau} \in W_{\tau} .
$$

For given $\left(u_{\tau, h}, \hat{u}_{h}\right) \in H_{\tau, h} \times \hat{U}_{h}$, we define $w_{\tau}=A_{\tau}^{-1}\left(B_{\tau} u_{\tau, h}+\hat{B}_{\tau} \hat{u}_{h}\right) \in W_{\tau}$ and the approximation $w_{\tau, h, p}=$ $A_{\tau, h, p}^{-1}\left(B_{\tau, h, p} u_{\tau, h}+\hat{B}_{\tau, h, p} \hat{u}_{h}\right) \in W_{\tau, h, p}$. Then we observe

$$
\left\|w_{\tau, h, p}-w_{\tau}\right\|_{V_{\tau}}=\inf _{v_{\tau, h, p} \in V_{\tau, h, p}}\left\|v_{\tau, h, p}-w_{\tau}\right\|_{V_{\tau}} .
$$

This yields

$$
\begin{aligned}
\sup _{v_{\tau, h, p} \in V_{\tau, h, p}} \frac{\left|\left\langle B_{\tau} u_{\tau, h}+\hat{B}_{\tau} \hat{u}_{h}, v_{\tau, h, p}\right\rangle\right|}{\left\|v_{\tau, h, p}\right\|_{V}} & =\left\|w_{\tau, h, p}\right\|_{V_{\tau}} \geq\left\|w_{\tau}\right\|_{V_{\tau}}-\left\|w_{\tau, h, p}-w_{\tau}\right\|_{V_{\tau}} \\
& \geq \beta_{0}\left\|w_{\tau}\right\|_{V_{\tau}}=\beta_{0} \sup _{v \in V} \frac{\left|\left\langle B_{\tau} u_{\tau, h}+B_{\tau} \hat{u}_{h}, v\right\rangle\right|}{\|v\|_{V}} .
\end{aligned}
$$

In some applications it may be required to choose $p \in \mathcal{P}$ depending of $\tau$ adaptively which can be achieved by a local variant of the greedy algorithm in [14].

Preconditioning. Although the discrete problem (6.2) is a well-defined symmetric positive definite system, the condition number depends on the mesh size. Thus, for an efficient solver a good preconditioner is required. To our knowledge, up to now for discontinuous Petrov-Galerkin methods only a one-level additive Schwarz preconditioner is discussed, see [3].

Here, we show that an efficient preconditioner for $\hat{S}_{h}$ can be constructed from an efficient preconditioner in $V_{h} \cap U$. For this purpose, assume that a subspace $U_{h} \subset U \cap V_{h}$ with trace space $\gamma_{\Gamma}\left(U_{h}\right)=\hat{U}_{h}$, a self-adjoint positive operator $A_{h}^{U} \in \mathcal{L}\left(U_{h}, U_{h}^{\prime}\right)$, and a self-adjoint preconditioner $G_{h} \in \mathcal{L}\left(U_{h}^{\prime}, U_{h}\right)$ exists.

Let $\gamma_{\Gamma, h} \in \mathcal{L}\left(U_{h}, \hat{U}_{h}\right)$ be the Galerkin approximation of $\left.\gamma_{\Gamma}\right|_{U_{h}}$ and define the corresponding skeleton preconditioner $\hat{G}_{h}=\gamma_{\Gamma, h} G_{h} \gamma_{\Gamma, h}^{\prime} \in \mathcal{L}\left(\hat{U}_{h}^{\prime}, \hat{U}_{h}\right)$ for $\hat{S}_{h}$. The following theorem shows that the skeleton preconditioner inherits its properties from the self-adjoint preconditioner, the inf-sup constant and suitable norm equivalences.

Theorem 6.4. Assume that $0<c_{A} \leq C_{A}$ and $0<c_{G} \leq C_{G}$ exists such that

$$
\begin{gathered}
c_{A}\left\langle A_{h}^{U} u_{h}, u_{h}\right\rangle \leq\left\|\gamma_{\Gamma} u_{h}\right\|_{\hat{U}}^{2} \leq C_{A}\left\langle A_{h}^{U} u_{h}, u_{h}\right\rangle, \\
c_{G}\left\langle A_{h}^{U} u_{h}, u_{h}\right\rangle \leq\left\langle A_{h}^{U} u_{h}, G_{h} A_{h}^{U} u_{h}\right\rangle \leq C_{G}\left\langle A_{h}^{U} u_{h}, u_{h}\right\rangle
\end{gathered}
$$

holds for $u_{h} \in U_{h}$. Then, with $c_{\hat{G}}=c_{G} c_{A} c_{S} \beta_{0}^{2}$ and $C_{\hat{G}}=C_{G} C_{A}$, we have

$$
c_{\hat{G}}\left\langle\hat{S}_{h} \hat{u}_{h}, \hat{u}_{h}\right\rangle \leq\left\langle\hat{S}_{h} \hat{u}_{h}, \hat{G}_{h} \hat{S}_{h} \hat{u}_{h}\right\rangle \leq C_{\hat{G}}\left\langle\hat{S}_{h} \hat{u}_{h}, \hat{u}_{h}\right\rangle, \quad \hat{u}_{h} \in \hat{U}_{h} .
$$

Proof. We proceed in two steps. Firstly, we bound $\left\langle\hat{S}_{h} \hat{u}_{h}, \hat{G}_{h} \hat{S}_{h} \hat{u}_{h}\right\rangle$ in terms of (6.4). Secondly, we show the spectral equivalence of $\hat{S}_{h}$ and $\hat{S}_{h} \gamma_{\Gamma, h} A_{h}^{-1} \gamma_{\Gamma, h}^{\prime} \hat{S}_{h}$. We have for $\hat{u}_{h} \in \hat{U}_{h}$

$$
\left\langle\hat{S}_{h} \hat{u}_{h}, \hat{G}_{h} \hat{S}_{h} \hat{u}_{h}\right\rangle=\left\langle A_{h}^{U}\left(A_{h}^{U}\right)^{-1} \gamma_{\Gamma, h}^{\prime} \hat{S}_{h} \hat{u}_{h}, G_{h} A_{h}^{U}\left(A_{h}^{U}\right)^{-1} \gamma_{\Gamma, h}^{\prime} \hat{S}_{h} \hat{u}_{h}\right\rangle
$$

and thus, using (6.4),

$$
c_{G}\left\langle\gamma_{\Gamma, h}^{\prime} \hat{S}_{h} \hat{u}_{h},\left(A_{h}^{U}\right)^{-1} \gamma_{\Gamma, h}^{\prime} \hat{S}_{h} \hat{u}_{h}\right\rangle \leq\left\langle\hat{S}_{h} \hat{u}_{h}, \hat{G}_{h} \hat{S}_{h} \hat{u}_{h}\right\rangle \leq C_{G}\left\langle\gamma_{\Gamma, h}^{\prime} \hat{S}_{h} \hat{u}_{h},\left(A_{h}^{U}\right)^{-1} \gamma_{\Gamma, h}^{\prime} \hat{S}_{h} \hat{u}_{h}\right\rangle .
$$


Introducing $\hat{A}_{h}^{U}=\left(\gamma_{\Gamma, h}\left(A_{h}^{U}\right)^{-1} \gamma_{\Gamma, h}^{\prime}\right)^{-1} \in \mathcal{L}\left(\hat{U}, \hat{U}^{\prime}\right)$, this equivalence reads as

$$
c_{G}\left\langle\hat{S}_{h} \hat{u}_{h},\left(\hat{A}_{h}^{U}\right)^{-1} \hat{S}_{h} \hat{u}_{h}\right\rangle \leq\left\langle\hat{S}_{h} \hat{u}_{h}, \hat{G}_{h} \hat{S}_{h} \hat{u}_{h}\right\rangle \leq C_{G}\left\langle\hat{S}_{h} \hat{u}_{h},\left(\hat{A}_{h}^{U}\right)^{-1} \hat{S}_{h} \hat{u}_{h}\right\rangle
$$

Thus it remains to show that $\hat{S}_{h}\left(\hat{A}_{h}^{U}\right)^{-1} \hat{S}_{h}$ is spectrally equivalent to $\hat{S}_{h}$.

For given $\hat{u}_{h} \in \hat{U}_{h}$ define $u_{h}=\left(A_{h}^{U}\right)^{-1} \gamma_{\Gamma, h}^{\prime} \hat{A}_{h}^{U} \hat{u}_{h}$ so that $\gamma_{\Gamma, h} u_{h}=\hat{u}_{h}$ and $\left\langle A_{h}^{U} u_{h}, u_{h}\right\rangle=\left\langle\hat{A}_{h}^{U} \hat{u}_{h}, \hat{u}_{h}\right\rangle$. Inserting these equalities in (6.3) yields

$$
c_{A}\left\langle\hat{A}_{h}^{U} \hat{u}_{h}, \hat{u}_{h}\right\rangle \leq\left\|\hat{u}_{h}\right\|_{\hat{U}}^{2} \leq C_{A}\left\langle\hat{A}_{h}^{U} \hat{u}_{h}, \hat{u}_{h}\right\rangle, \quad \hat{u}_{h} \in \hat{U}_{h} .
$$

Together with the spectral bound (6.1), we obtain

$$
c_{A} c_{S} \beta_{0}^{2}\left\langle\hat{A}_{h}^{U} \hat{u}_{h}, \hat{u}_{h}\right\rangle \leq\left\langle\hat{S}_{h} \hat{u}_{h}, \hat{u}_{h}\right\rangle \leq C_{A}\left\langle\hat{A}_{h}^{U} \hat{u}_{h}, \hat{u}_{h}\right\rangle, \quad \hat{u}_{h} \in \hat{U}_{h}
$$

or equivalently

$$
c_{A} c_{S} \beta_{0}^{2}\left\langle\hat{F}_{h}, \hat{S}_{h}^{-1} \hat{F}_{h}\right\rangle \leq\left\langle\hat{F}_{h},\left(\hat{A}_{h}^{U}\right)^{-1} \hat{F}_{h}\right\rangle \leq C_{A}\left\langle\hat{F}_{h}, \hat{S}_{h}^{-1} \hat{F}_{h}\right\rangle, \quad \hat{F}_{h} \in \hat{U}_{h}^{\prime}
$$

The choice $\hat{F}_{h}=\hat{S}_{h} \hat{u}_{h}$ now gives

$$
c_{A} c_{S} \beta_{0}^{2}\left\langle\hat{S}_{h} \hat{u}_{h}, \hat{u}_{h}\right\rangle \leq\left\langle\hat{S}_{h} \hat{u}_{h},\left(\hat{A}_{h}^{U}\right)^{-1} \hat{S}_{h} \hat{u}_{h}\right\rangle \leq C_{A}\left\langle\hat{S}_{h} \hat{u}_{h}, \hat{u}_{h}\right\rangle
$$

and in combination with (6.5) the assertion is verified.

We shortly comment on a suitable construction of $U_{h}$ satisfying (6.3). Let $\hat{P}_{\Gamma} \in \mathcal{L}\left(U, \mathcal{N}\left(\gamma_{\Gamma}\right)\right)$ be the orthogonal projection to $\mathcal{N}\left(\gamma_{\Gamma}\right)$. Then, we have

$$
\left\|\gamma_{\Gamma} u_{h}\right\|_{\hat{U}}=\inf _{v \in \mathcal{N}\left(\gamma_{\Gamma}\right)}\left\|u_{h}-v\right\|_{U}=\left\|u_{h}-\hat{P}_{\Gamma} u_{h}\right\|_{U}
$$

This shows that choosing $U_{h}=\left(\operatorname{id}_{U_{h}}-\hat{P}_{\Gamma, h}\right)\left(U \cap V_{h}\right)$ with the orthogonal projection $\hat{P}_{\Gamma, h} \in \mathcal{L}\left(U \cap V_{h}, \mathcal{N}\left(\gamma_{\Gamma, h}\right)\right)$ gives (6.3) with $c_{A} \in(0,1)$ close to 1 and $C_{A}=1$, if $V_{h}$ is fine enough and if the operator $A_{h}^{U}$ is given by $\left\langle A_{h}^{U} u_{h}, \tilde{u}_{h}\right\rangle=\left(u_{h}, \tilde{u}_{h}\right)_{H}+\left(L u_{h}, L \tilde{u}_{h}\right)_{H}$.

Error control. The condition $\hat{U}_{h} \subset \gamma_{\Gamma}\left(V_{h}\right)$ allows for a local reconstruction of the discrete skeleton solution $\hat{u}_{h}^{\text {sol }} \in \hat{U}_{h}$ in $U$ by determining $v_{h}^{\text {sol }} \in V_{h}$ such that $\gamma_{\Gamma} v_{h}^{\text {sol }}=\hat{u}_{h}^{\text {sol }}$. Then, we have $v_{h}^{\text {sol }} \in U \cap V_{h}$ and the error bound

$$
\left\|\hat{u}^{\mathrm{sol}}-\hat{u}_{h}^{\mathrm{sol}}\right\|_{\hat{U}} \leq\left\|u^{\mathrm{sol}}-v_{h}^{\mathrm{sol}}\right\|_{U} \leq \sqrt{1+C_{L}^{2}}\left\|L\left(u^{\mathrm{sol}}-v_{h}^{\mathrm{sol}}\right)\right\|_{H}=\sqrt{1+C_{L}^{2}}\left\|f-L v_{h}^{\mathrm{sol}}\right\|_{H}
$$

In the special case $\hat{U}_{h}=\gamma_{\Gamma}\left(V_{h}\right)$ and $\mathcal{N}\left(\gamma_{\Gamma}\right)=\{0\}$ the reconstruction is unique. Then, the error bound is minimal for the result of the standard least squares method in $U_{h}=U \cap V_{h}$, i.e., by computing a minimizer of $R\left(v_{h}\right)=\frac{1}{2}\left\|f-L v_{h}\right\|_{H}^{2}$.

Better results can be expected if $\mathcal{N}\left(\gamma_{\Gamma}\right) \neq\{0\}$, where the lifting $v_{\tau, h}^{\text {sol }}$ with $\gamma_{\Gamma} v_{h}^{\text {sol }}=\hat{u}_{h}^{\text {sol }}$ is not unique, i.e.,

$$
v_{\tau, h}^{\mathrm{sol}} \in A_{\tau, h}^{-1} \gamma_{\tau, h}^{\prime}\left(\gamma_{\tau, h} A_{\tau, h}^{-1} \gamma_{\tau, h}^{\prime}\right)^{-1} \hat{u}_{h}^{\mathrm{sol}}+\mathcal{N}\left(\gamma_{\tau}\right)
$$

Uniqueness can be achieved by the additional condition $\left(L v_{\tau, h}^{\text {sol }}-f, v_{h}\right)_{H_{\tau}}=0$ for $v_{h} \in \mathcal{N}\left(\gamma_{\tau}\right)$. 

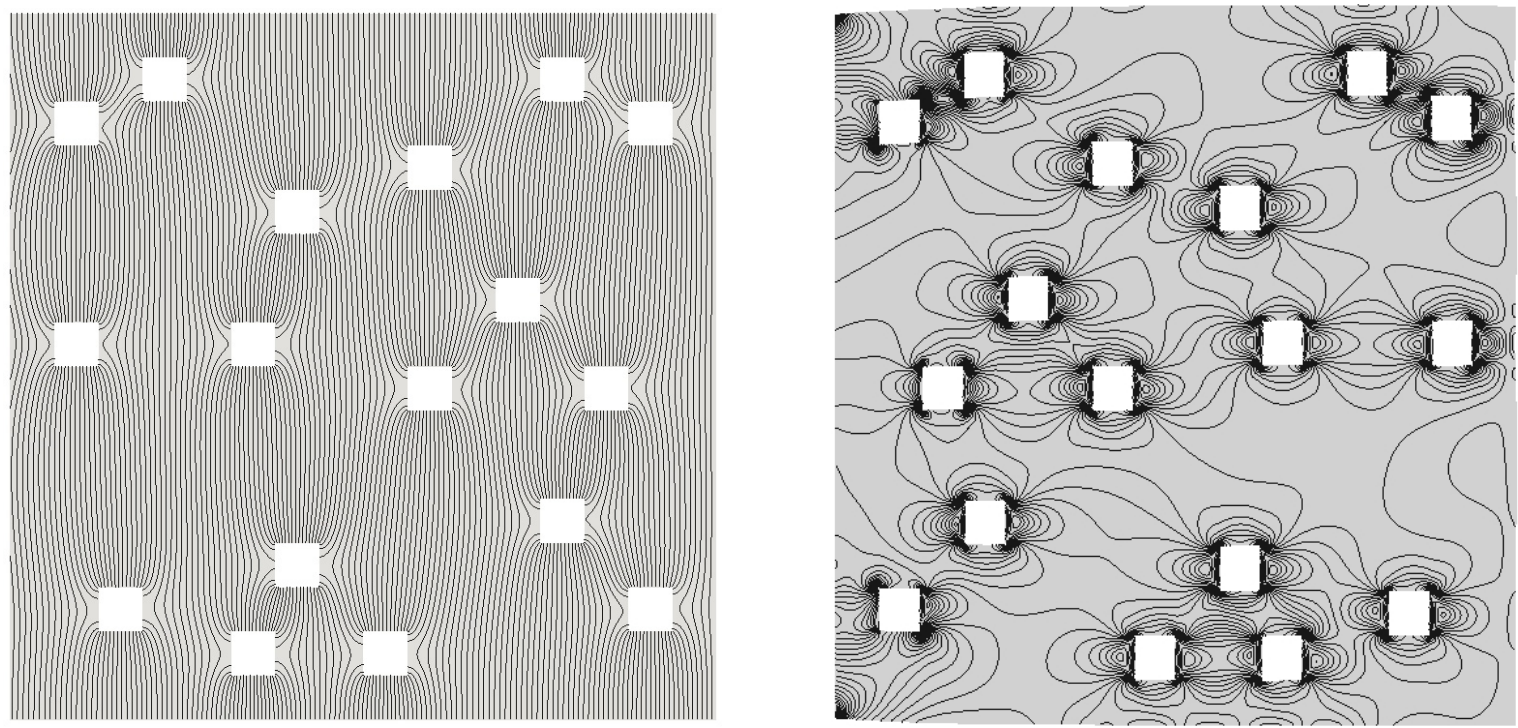

FiguRE 1. Isolines of $p$ for a diffusion problem with homogeneous Dirichlet boundary values on the top boundary $\left(x_{2}=1\right)$, Neumann boundary values $\sigma \cdot n=1$ on the bottom boundary $\left(x_{2}=0\right)$, and homogeneous Neumann boundary values else (left), and isolines of $|\sigma|$ for nearly incompressible linear elasticity with homogeneous Dirichlet boundary values on the bottom boundary $\left(x_{2}=0\right)$, a pressure load for $x_{2}=1$, and homogeneous Neumann boundary values else (right).

\section{NUMERICAL EXAMPLES}

As a proof of concept, we consider two simple examples. In both cases we test the convergence of the discretization by computing the error bound (6.7), and we test the convergence of the skeleton preconditioner $\hat{G}_{h}$.

The first example is a diffusion problem $-\Delta p=0$ in $\Omega \subset(0,1)^{2}$ with impenetrable obstacles in the domain, see Figure 1 for the geometry and boundary conditions. Let $U_{h}=\mathrm{RT}_{0, \partial \Omega_{\mathrm{N}}}\left(\mathcal{T}_{h}\right) \times \mathrm{S}_{1, \partial \Omega_{\mathrm{D}}}\left(\mathcal{T}_{h}\right)$ be the lowest order finite element space, $\hat{U}_{h}=\gamma_{\Gamma}\left(U_{h}\right)$ its trace space, $H_{K}=\mathbb{P}_{0}(K)^{2} \times \mathbb{P}_{0}(K)$, and $V_{K}=\mathbb{P}_{1}(K)^{2} \times \mathbb{P}_{1}(K)$. For preconditioning, we choose

$$
\left\langle A_{h}^{U}\left(\left(\sigma_{h}, p_{h}\right),\left(\tilde{\sigma}_{h}, \tilde{p}_{h}\right)\right)=\left(\sigma_{h}, \tilde{\sigma}_{h}\right)_{\Omega}+\left(\nabla \cdot \sigma_{h}, \nabla \cdot \tilde{\sigma}_{h}\right)_{\Omega}+\left(p_{h}, \tilde{p}_{h}\right)_{\Omega}+\left(\nabla p_{h}, \nabla \tilde{p}_{h}\right)_{\Omega}, \quad\left(\sigma_{h}, p_{h}\right),\left(\tilde{\sigma}_{h}, \tilde{p}_{h}\right) \in U_{h} .\right.
$$

In the first component, $G_{h}$ is a $\mathrm{H}(\operatorname{div}, \Omega)$ multigrid preconditioner with hybrid smoothing [24], and in the second component we use a parallel damped block Jacobi smoothing and local Gauß-Seidel smoothing on each processor [32]. As stopping criteria for the parallel Krylov iteration with $\mathrm{V}(2,2)$-multigrid preconditioning, we use a residual reduction by the factor $10^{-12}$. The results in the upper part of Table 1 show asymptotically the expected convergence rate $O\left(h^{\frac{2}{3}}\right)$ for the lowest order approximation and mesh independent convergence for the multigrid preconditioner.

In our second example, we test the robustness of the discrete scheme for nearly incompressible 2D linear elasticity $-\operatorname{div} \mathcal{C} \varepsilon(u)=0$ with constant pressure load $\sigma n=-p_{0} n$ on $\partial \Omega_{\mathrm{N}} \subset \partial \Omega$, see Figure 1 . Here, we use $H_{K}=\mathbb{P}_{0}(K)_{\text {sym }}^{2 \times 2} \times \mathbb{P}_{0}(K)^{2}$ and $V_{K}=\mathbb{P}_{1}(K)_{\text {sym }}^{2 \times 2} \times \mathbb{P}_{1}(K)^{2}$, and a $\mathrm{V}(4,4)$-multigrid cycle is applied. The results in the lower part of Table 1 show robustness of the approximation and for the multigrid preconditioner in the incompressible limit.

The transfer to more challenging problems requires the proper choice of $A_{h}^{U}$ in $U_{h}=V_{h} \cap U$, an efficient preconditioner $G_{h}$, and for the error control a suitable reconstruction operator. 
TABLE 1. Numerical results for the diffusion problem and for linear elasticity. The reconstruction in (6.7) is determined by the evaluation of $\hat{u}^{\text {sol }}$ at the nodal points of $U_{h}$, and the error is estimated by $\rho_{h}=\|(\sigma+\nabla p, \nabla \cdot \sigma-f)\|_{\Omega}$ for the diffusion problem and by $\rho_{h}=\left\|\left(\mathcal{C}^{-1} \sigma-\mathrm{D} u, \operatorname{div} \sigma+f\right)\right\|_{\Omega}$ for elasticity.

\begin{tabular}{lcccccccc}
\hline & number of triangles & 1912 & 7648 & 30592 & 122368 & 489472 & 1957888 & 7831552 \\
\hline diffusion & $\operatorname{dim}\left(\hat{U}_{h}\right)$ & 4072 & 15808 & 62224 & 246832 & 983152 & 3924208 & 15679984 \\
& $\rho_{h}$ & 0.24044 & 0.15507 & 0.09852 & 0.06227 & 0.03928 & 0.02475 & 0.01560 \\
& $\log _{2} \frac{\rho_{2 h}}{\rho_{h}}$ & & 0.633 & 0.654 & 0.662 & 0.665 & 0.666 & 0.666 \\
& multigrid steps & & 19 & 20 & 20 & 21 & 21 & 21 \\
\hline elasticity & $\operatorname{dim}\left(\hat{U}_{h}\right)$ & 8144 & 31616 & 124448 & 493664 & 1966304 & 7848416 \\
$\nu=0.49$ & $\rho_{h}$ & 0.010224 & 0.006978 & 0.004593 & 0.003028 & 0.002010 & 0.0013434 \\
& $\log _{2} \frac{\rho_{2 h}}{\rho_{h}}$ & & 0.551 & 0.60333 & 0.601 & 0.591 & 0.582 \\
$\nu=0.499$ & $\rho_{h}$ & 0.010170 & 0.006941 & 0.004569 & 0.003014 & 0.002002 & 0.001338 \\
& $\operatorname{multigrid~steps}$ & & 0.550 & 0.603 & 0.600 & 0.590 & 0.580 \\
& $\log _{2} \frac{\rho_{2 h}}{\rho_{h}}$ & & 32 & 33 & 33 & 34 & 36 \\
$\nu=0.4999$ & $\rho_{h}$ & 0.010164 & 0.006938 & 0.004567 & 0.003012 & 0.002001 & 0.001338 \\
& $\rho_{h}$ & & 0.550 & 0.603 & 0.600 & 0.590 & 0.580 \\
& $\log _{2} \frac{\rho_{2 h}}{\rho_{h}}$ & 33 & 33 & 33 & 34 & 36 \\
\hline
\end{tabular}

Concluding remarks. Robust operator estimates and, as a consequence, a priori estimates in operator depending norms are essential for the design of robust discretization methods and robust numerical solvers. Here we have shown that for representative problem classes suitable stability estimates can be provided leading to convergence estimates of discontinuous Petrov-Galerkin methods. The efficiency of the preconditioning is demonstrated for diffusion and elasticity. Further numerical results for the Poisson problem [15], for the transport equation [13], for convection diffusion problems [12,18], for the Helmholtz problem [16], and for the Stokes problem [30] underline the flexibility and efficiency of discontinuous Petrov-Galerkin methods. Nevertheless, to exploit the full potential of a Petrov-Galerkin approach, further research is required to identify the optimal balance of local approximations and skeleton discretizations.

\section{REFERENCES}

[1] J.H. Adler, J. Brannick, C. Liu, T. Manteuffel and L. Zikatanov, First-order system least squares and the energetic variational approach for two-phase flow. J. Comput. Phys. 230 (2011) 6647-6663.

[2] J.H. Adler, T.A. Manteuffel, S.F. McCormick, J.W. Nolting, J.W. Ruge and L. Tang, Efficiency based adaptive local refinement for first-order system least-squares formulations. SIAM J. Sci. Comput. 33 (2011) 1-24.

[3] A. Barker, S. Brenner, E.-H. Park and L-Y. Sung, A one-level additive schwarz preconditioner for a discontinuous petrovgalerkin method. Preprint arXiv:1212.2645 (2012). To appear in the Proceeding of DD21.

[4] P.B. Bochev and M.D. Gunzburger, Finite element methods of least-squares type. SIAM Rev. 40 (1998) $789-837$.

[5] P.B. Bochev and M.D. Gunzburger, Least-Squares Finite Element Methods, vol. 166 of Appl. Math. Sci. Springer, New York (2009).

[6] D. Braess, Finite Elements. Theory, fast solvers, and applications in solid mechaics. 3th ed. Cambridge University Press (2007).

[7] J.H. Bramble, R.D. Lazarov and J.E. Pasciak, A least-squares approach based on a discrete minus one inner product for first order systems. Math. Comput. 66 (1997) 935-955.

[8] F. Brezzi and M. Fortin, Mixed and Hybrid Finite Element Methods. Springer (1991).

[9] T. Bui-Thanh, L. Demkowicz and O. Ghattas, A Unified Discontinuous Petrov-Galerkin Method and its Analysis for Friedrichs' Systems. SIAM J. Numer. Anal. 51 (2013) 1933-1956.

[10] A. Buffa and P. Monk, Error estimates for the ultra weak variational formulation of the Helmholtz equation. Math. Model. Numer. Anal. 42 (2008) 925-940.

[11] Z. Cai, R. Lazarov, T.A. Manteuffel and S.F. McCormick, First-Order System Least Squares for Second-Order Partial Differential Equations: Part I. SIAM J. Numer. Anal. 31 (1994) 1785-1799. 
[12] J. Chan, L. Demkowicz and N. Heuer, Robust DPG method for convection-dominated diffusion problems II: Natural inflow condition. Comput. Math. Appl. 67 (2014) 771-795.

[13] W. Dahmen, C. Huang, C. Schwab and G. Welper, Adaptive Petrov-Galerkin methods for first order transport equations. SIAM J. Numer. Anal. 50 (2012) 2420-2445.

[14] W. Dahmen, C. Plesken and G. Welper, Double greedy algorithms: reduced basis methods for transport dominated problems (2013). Preprint arXiv:1302.5072.

[15] L. Demkowicz and J. Gopalakrishnan, Analysis of the DPG method for the Poisson equation. SIAM J. Numer. Anal. 49 (2011) 1788-1809.

[16] L. Demkowicz, J. Gopalakrishnan, I. Muga and J. Zitelli, Wavenumber explicit analysis for a DPG method for the multidimensional Helmholtz equation. Comput. Methods Appl. Mech. Engrg. 213 (2012) 126-138.

[17] L. Demkowicz, J. Gopalakrishnan and A.H. Niemi, A class of discontinuous Petrov-Galerkin methods. Part III: Adaptivity. Appl. Numer. Math. 62 (2012) 396-427.

[18] L. Demkowicz and N. Heuer, Robust DPG method for convection-dominated diffusion problems. SIAM J. Numer. Anal. 51 (2013) 2514-2537.

[19] S. Esterhazy and J.M. Melenk, On stability of discretizations of the Helmholtz equation, in Numerical Analysis of Multiscale Problems, vol. 83 of Lect. Notes Comput. Sci. Engrg. Springer, Berlin (2012) 285-324.

[20] J. Gopalakrishnan and W. Qiu, An analysis of the practical DPG method. Math. Comput. (2013).

[21] I. Herrera, Trefftz method: A general theory. Numer. Methods Partial Differ. Eqs. 16 (2000) 561-580.

[22] J.J. Heys, E. Lee, T.A. Manteuffel, S.F. Mccormick and J.W. Ruge, Enhanced mass conservation in least-squares methods for Navier-Stokes equations. SIAM J. Sci. Comput. 31 (2009) 2303-2321.

[23] R. Hiptmair, A. Moiola and I. Perugia, Stability results for the time-harmonic Maxwell equations with impedance boundary conditions. Math. Models Methods Appl. Sci. 21 (2011) 2263-2287.

[24] R. Hiptmair and J. Xu, Nodal auxiliary space preconditioning in $\mathrm{H}(\mathrm{curl})$ and $\mathrm{H}(\mathrm{div})$ spaces. SIAM J. Numer. Anal. 45 (2007) 2483-2509.

[25] B.N. Khoromskij and G. Wittum, Numerical solution of elliptic differential equations by reduction to the interface. Berlin, Springer (2004).

[26] W. Krendl, V. Simoncini and W. Zulehner, Stability Estimates and Structural Spectral Properties of Saddle Point Problems. Numer. Math. 124 (2013) 183-213.

[27] U. Langer, G. Of, O. Steinbach and W. Zulehner, Inexact data-sparse boundary element tearing and interconnecting methods. SIAM J. Sci. Comput. 29 (2007) 290-314.

[28] J.M. Melenk, On generalized finite element methods. Ph.D. thesis, University of Maryland (1995).

[29] A. Moiola, Trefftz-Discontinuous Galerkin Methods for Time-Harmonic Wave Problems. Ph.D. thesis, ETH Zürich (2011).

[30] N. Roberts, T. Bui-Thanh and L. Demkowicz. The DPG method for the Stokes problem ICES Report (2012) 12-22.

[31] D.B. Szyld, The many proofs of an identity on the norm of oblique projections. Numer. Algorithms 42 (2006) $309-323$.

[32] C. Wieners, A geometric data structure for parallel finite elements and the application to multigrid methods with block smoothing. Comput. Visual. Sci. 13 (2010) 161-175.

[33] J. Xu and L. Zikatanov, Some observations on Babuška and Brezzi theories. Numer. Math. 94 (2003) $195-202$.

[34] J. Zitelli, I. Muga, L. Demkowicz, J. Gopalakrishnan, D. Pardo and V. Calo, A class of discontinuous Petrov-Galerkin methods. Part IV: Wave propagation. J. Comput. Phys. 230 (2011) 2406-2432. 